\title{
Strong Gravitational Lensing by Wave Dark Matter Halos
}

\author{
Antonio Herrera-Martín ${ }^{1,2}$ (10 , Martin Hendry $^{1}$ (D) , Alma X. Gonzalez-Morales ${ }^{3,4}$ (D) , and L. Arturo Ureña-López ${ }^{4}$ (1) \\ ${ }^{1}$ SUPA, University of Glasgow, Glasgow, G12 8QQ, UK; a.herrera-martin.1@ research.gla.ac.uk, \\ antonio.herreramartin@canterbury.ac.nz,martin.hendry@glasgow.ac.uk \\ ${ }^{2}$ School of Physical and Chemical Sciences, University of Canterbury Christchurch, New Zealand \\ ${ }^{3}$ Consejo Nacional de Ciencia y Tecnología, Av. Insurgentes Sur 1582. Colonia Crédito Constructor, \\ Del. Benito Jurez C.P. 03940, México D.F. México; alma.gonzalez@ fisica.ugto.mx \\ ${ }^{4}$ Departamento de Física, DCI, Campus León, Universidad de Guanajuato, 37150, León, Guanajuato, México; lurena@ugto.mx \\ Received 2017 September 7; revised 2018 December 20; accepted 2018 December 24; published 2019 February 6
}

\begin{abstract}
Wave dark matter (WaveDM) has recently gained attention as a viable candidate to account for the dark matter content of the universe. In this paper we explore the extent to which, and under what conditions, dark matter halos in this model are able to reproduce strong-lensing systems. First, we explore analytically the lensing properties of the model, finding that a pure WaveDM density profile, the soliton profile, produces a weaker lensing effect than similar cored profiles. Then, we analyze models with a soliton embedded within a Navarro, Frenk, and White (NFW) profile, as has been found in numerical simulations of structure formation. We use a benchmark model with a boson mass of $m_{a}=10^{-22} \mathrm{eV}$, for which we see that there is a bimodality in the contribution of the external NFW part of the profile, and some of the free parameters associated with it are not well constrained. We find that for configurations with boson masses $10^{-23}$ to $10^{-22} \mathrm{eV}$, a range of masses preferred by dwarf galaxy kinematics, the soliton profile alone can fit the data, but its size is incompatible with the luminous extent of the lens galaxies. Likewise, boson masses of the order of $10^{-21} \mathrm{eV}$, which would be consistent with Ly $\alpha$ constraints and consist of more compact soliton configurations, necessarily require the NFW part in order to reproduce the observed Einstein radii. We then conclude that lens systems impose a conservative lower bound $m_{a}>10^{-24} \mathrm{eV}$ and that the NFW envelope around the soliton must be present to satisfy the observational requirements.
\end{abstract}

Key words: dark matter - Galaxy: halo - gravitational lensing: strong

\section{Introduction}

The $\Lambda$ CDM model is the most successful theoretical framework in modern cosmology to explain the process of structure formation in the universe on large scales. This model requires the existence of a cold dark matter $(\mathrm{CDM})$ component that composes $26 \%$ of the total energy budget, which is best described by a nonrelativistic (cold) and noninteracting fluid (see Planck Collaboration et al. 2016).

One of the main predictions from only CDM simulations of structure formation is the appearance of universal cuspy density profiles for the galaxy halos, with the Navarro, Frenk, and White (NFW) profile the one most used to describe CDM (see Navarro et al. 1997). Despite the successes of CDM at large scales, there are some open questions regarding the observations on galactic scales, such as the "missing satellite problem," the "cusp core problem," and the "too-big-to-fail problem" (e.g., Burkert 1995; Klypin et al. 1999; de Blok 2010; Boylan-Kolchin et al. 2011; Oh et al. 2011; Sawala et al. 2011; Maccio et al. 2012; see also Bullock \& BoylanKolchin 2017 for a recent review). These refer to both theoretical and observational questions on how the interplay of CDM and baryons leads to the shapes, inner density profiles, and abundance of the DM structure at subgalactic scales. This also opens the question weather such observables, or others, can be used to learn more about the nature of dark matter (DM). This has lead us to explore the viability of other DM candidates. Actually there is a wide range of DM proposals, such as self-interacting DM

Original content from this work may be used under the terms of the Creative Commons Attribution 3.0 licence. Any further distribution of this work must maintain attribution to the author(s) and the title of the work, journal citation and DOI. (see Kaplinghat et al. 2016), warm DM (see Gonzalez-Samaniego et al. 2016; Adhikari et al. 2017), axion/scalar or wave DM (WaveDM; e.g., Goodman 2000; Hu et al. 2000; Matos et al. 2000; Matos \& Urena-Lopez 2001; Böhmer \& Harko 2007; Robles \& Matos 2013; Schive et al. 2014a), and other specifications of the nature of DM particles, which can actually be described in a more general effective theory (e.g., Cyr-Racine et al. 2016).

In this paper, our approach is to describe the DM as an axion/ scalar field that we will refer to as the WaveDM model (also referred to sometimes as scalar field DM or SFDM, ultralight axion-like DM, fuzzy DM, etc.). This type of model has been investigated by several other authors (e.g., Goodman 2000; Hu et al. 2000; Matos et al. 2000; Matos \& Urena-Lopez 2001; Böhmer \& Harko 2007; Suárez et al. 2014; Lee 2018) and has been found to be able to reproduce the success of the $\Lambda \mathrm{CDM}$ model on cosmological scales, but it predicts a natural cutoff on the mass power spectrum of linear perturbations that could help to alleviate some of the low-scale issues of CDM (e.g., Hu et al. 2000; Matos \& Urena-Lopez 2001; Hlozek et al. 2015; UreñaLópez \& Gonzalez-Morales 2016). Interestingly enough, all cosmological effects are directly related to a single parameter, which is the boson mass, $m_{a}$, of the scalar field particle, although extra observational effects may arise from quartic self-interactions (e.g., Linares Cedeño et al. 2017; Schive \& Chiueh 2018; Zhang $\&$ Chiueh 2017a, 2017b). Based on considering the cutoff of the mass power spectrum, the halo mass function, the reionization time, or the Ly $\alpha$ forest, the most up-to-date constraints suggest that the boson mass must satisfy $m_{a}>1 \times 10^{-21} \mathrm{eV}$ (see Armengaud et al. 2017; Iršič et al. 2017).

However, the nonlinear process of structure formation under the SFDM hypothesis does not depend on only a single parameter, but instead it is required to take into account at least 
a second parameter. This fact is indeed considered in many recent studies that try to put constraints on the WaveDM parameters with data coming from, for instance, satellite galaxies in the Milky Way (e.g., Schive et al. 2014a, 2014b; Chen et al. 2017; González-Morales et al. 2017; Bernal et al. 2018). The aforementioned studies consider that galaxies are described by a solitonic core with a negligible self-interaction. The soliton solution is just the ground state of the so-called Schrodinger-Poisson (SP) system of equations (Ruffini \& Bonazzola 1969; Guzman \& Urena-Lopez 2004), and its wavelike properties provide stability against gravitational collapseopening the possibility of naturally supported, cored halos. The full prescription of the WaveDM profile requires specification of the boson mass $m_{a}$, together with one of its structure parameters, which can be taken to be either the central density or the scale radius, while the other is determined by the relation

$$
\frac{\rho_{s}}{M_{\odot} \mathrm{pc}^{-3}}=2.4 \times 10^{12}\left(\frac{r_{s}}{\mathrm{pc}}\right)^{-4}\left(\frac{m_{a}}{10^{-22} \mathrm{eV}}\right)^{-2} \text {. }
$$

The boson mass $m_{a}$ is expected to be a fundamental parameter with a single value for all galaxies, while the other two parameters may take values that differ from galaxy to galaxy. Hence, it is necessary to think more carefully if we are to obtain meaningful constraints on the boson mass. More specifically, if we consider the boson mass as a universal parameter, on the same footing as any other cosmological parameter, we should certainly be able to use statistical analysis of galaxy data to constrain which values are permitted, as has been proposed in González-Morales et al. (2013) and Diez-Tejedor et al. (2014) and more recently carried out in Chen et al. (2017) and González-Morales et al. (2017). However, in general, we may be unable to assert whether there is one single value of $m_{a}$ that is suitable to satisfy all the possible constraints. For this purpose, in this paper we have selected gravitational lensing as a possible additional tool to study or assess the viability of the WaveDM profile whose parameters are subjected to the constraint in Equation (1).

Gravitational lensing (strong and weak) has become a powerful astrophysical tool for the study of the background cosmology, structure, and substructures of galactic halos (see Schneider et al. 1999; Koopmans 2005; Bolton et al. 2006; Cao et al. 2015, 2016). In particular, it is possible to extract important information from the stellar kinematics and geometry of strong-lensed systems. Furthermore, particular cosmologies where dark energy is not a cosmological constant can be tested by fitting the observed critical lines, Einstein angle, and stellar dynamics, given a suitable lens model for the observed systems (see, e.g., Futamase \& Yoshida 2001; Sereno 2002; Grillo et al. 2008; Newton et al. 2011). As the geometry of the lensing system can be obtained from image astrometry, it also is a helpful probe for the Hubble parameter and the dark energy contents (Mitchell et al. 2005; Biesiada et al. 2010), whereas at the same time it gives information about the structure and formation of early-type galaxies (Koopmans et al. 2006; Gavazzi et al. 2007).

There are different procedures to extract particular parameters, like the Einstein angle, from the lensing geometry in combination with velocity dispersions from stellar dynamics (Bolton et al. 2008b; Auger et al. 2010), where the latter can be obtained along with redshifts from spectroscopic imaging (Kochanek 1991; Ofek et al. 2003, 2006; Bolton et al. 2006; Cao \& Zhu 2012). When assuming a particular lens model, it is common, as a first approach, to describe strong lenses using an axially symmetric model, the most popular being the singular isothermal sphere (SIS; Schneider et al. 1999; Mollerach \& Roulet 2002; Auger et al. 2009; Cao et al. 2012). Sometimes, however, there are deviations from the SIS model, and the singular isothermal ellipsoid (SIE) is used instead as a nonaxially symmetric extension (Gavazzi et al. 2007; Cao et al. 2015). By assuming these models, which consider the total mass distribution of the lens, there is some freedom to test the cosmological parameters (Sereno 2002; Cao et al. 2016; Jie et al. 2016). On the other hand, using a fixed cosmology allows one to obtain information of the physical parameters regarding the structure of individual galaxies or clusters of galaxies (Futamase \& Yoshida 2001; Sereno 2002; Grillo et al. 2008). Furthermore, using information obtained from the strong-lensed galaxies, it is possible to test the gravitational weak field, through the post-Newtonian parameter $\gamma$, where the parameterization of the profiles to describe is sensitive to the total mass distribution. This can be used to test the validity of general relativity on large scales. Recently, it has been found that for at least $\sim 2 \mathrm{kpc}$, the lensing galaxy profiles agree with general relativity (see, e.g., Cao et al. 2017; Collett et al. 2018).

For the description of lensing systems, several mass distribution profiles exist that have been successful enough to represent observed data; the most popular, which include the SIS and SIE (see, e.g., Keeton 2001), follow a power-law distribution. These are very successful in describing the observed data for lensing geometry and stellar dynamics when considering the total matter contents (luminous+DM; Bolton et al. 2008a, 2008b; Auger et al. 2009; Cao et al. 2012, 2015; Suyu et al. 2014). It is important to stress that these modeling choices do not impact the estimated Einstein angle of the lens. Nevertheless, these modeling choices do not explicitly give much information about the properties of the internal structure of the DM distribution in the galaxies. For this reason, other composite models where the baryonic matter and the DM are treated separately are also used (e.g., Navarro et al. 1997; Park \& Ferguson 2003; Suyu et al. 2014). The luminous part, which contains the baryonic matter, is described by an SIS, but it is common to use Sérsic or more general power-law luminosity profiles (Cardone 2004; Cao et al. 2016). The most popular way to describe the DM halo of a galaxy is the NFW density profile (Bartelmann 1996; Wright \& Brainerd 2000), which introduces a family of generalized NFW profiles (Wyithe et al. 2001). Other popular profiles are the Burkert profile (Burkert 1995; Park \& Ferguson 2003) and cuspy halo models (Keeton 2001; Muñoz et al. 2001). It has been shown that the NFW profile correctly describes the observed lensing signal in large samples of galaxies (Gavazzi et al. 2007) and clusters of galaxies (Niikura et al. 2015).

Since the WaveDM model is considered a feasible candidate for DM, in this work we study the behavior of, and put constraints on, a WaveDM type of profile acting as a single galactic gravitational lens, and we obtain the conditions under which the profile will be able to produce strong lensing. As we shall see, the WaveDM profile consists of a solitonic core plus a tail in the outer parts that follows the prescription of an NFW profile, whose properties are closely interlinked by their matching conditions such that a soliton is always present in the center of the DM halo. In this respect, we are not interested in the individual properties of the single profiles (soliton vs. NFW), but rather in the conditions under which the complete WaveDM profile could be consistent with lensing data.

The remainder of this paper is organized as follows. The basic lensing equations for any given density profile are described in Section 2, where we also introduce the explicit 
lensing expressions for the particular case of the WaveDM profile. In Section 3 we describe our statistical analysis and present the results arising from the comparison of the WaveDM model predictions with selected data from the SLACS catalog. Finally, the general conclusions are presented in Section 4. Some analytic solutions of the lens equations used in the text are shown in the Appendix.

\section{Gravitational Lensing with a $\psi \mathrm{DM}$ Profile}

\subsection{General Lensing Equations}

One of the main predictions from Einstein's general relativity is the bending of light as it passes close to a massive body. The deflection angle produced by this effect depends on the mass of the deflector, which then acts like a lens. This deflector may be approximated by a point-like mass, like in the case of a star, but for more massive objects like galaxies it is better to represent them as extended masses that are described by their density profiles. For the purposes of this work, we shall consider galactic lenses, and therefore the density profile described in this section will be representing one galaxy acting as a single lens.

The simplest type of lens is a system with a point mass $M$ located close to the line of sight to a luminous source $S$. Due to the gravitational field of the point mass, a light ray is deflected in its path to the observer; this is described by the lens equation in the thin-lens approximation. The same approximation also holds for a mass distribution, in which case the lens equation is (Mollerach \& Roulet 2002)

$$
\beta=\theta-\frac{m(\theta)}{\pi \Sigma_{\mathrm{cr}} D_{\mathrm{OL}}^{2} \theta},
$$

which relates the (unobservable) angle between the line of sight and the path from the observer to the actual position of the source, $\beta$, and to the apparent position of the source (the image), $\theta$, and the mass distribution that is causing the lensing $m(\theta)$. Also, $D_{\mathrm{OL}}$ is the angular distance from the observer to the lens, which we denote by the subindex (OL). Here we have assumed that $m(\theta)$ is the projected mass enclosed in a circle of radius $\xi \equiv D_{\mathrm{OL}} \theta$; more explicitly, we can write

$$
m(\xi)=2 \pi \int_{0}^{\xi} d \hat{\xi} \hat{\xi} \Sigma(\hat{\xi}) .
$$

The projected surface mass density $\Sigma(\xi)$ can be calculated directly from the (spherically symmetric) density profile $\rho(r)$ of the lensing object as

$$
\Sigma(\xi)=2 \int_{0}^{z_{\max }} d z \rho(z, \xi),
$$

where $z \equiv \sqrt{r^{2}-\xi^{2}}$ is a coordinate orthogonal to the line of sight, so that $0 \leqslant \xi \leqslant r$. If the lens system has a finite radius $r_{\max }$, then $z_{\max }=\sqrt{r_{\max }^{2}-\xi}$; otherwise, we can put $z_{\max } \rightarrow \infty$ in the integral given by Equation 3(b).

Let us consider the case in which the density profile $\rho(r)$ has a characteristic density $\rho_{s}$ and a characteristic radius $r_{s}$, such that $\rho(r)=\rho_{s} f\left(r / r_{s}\right)$, where $f$ is the function that accounts for the shape of the profile. We can then write Equation (2) in the dimensionless form

$$
\beta_{*}\left(\theta_{*}\right)=\theta_{*}-\lambda \frac{m_{*}\left(\theta_{*}\right)}{\theta_{*}},
$$

where the different distances are normalized in terms of $r_{s}$ : $\beta_{*}=D_{\mathrm{OL}} \beta / r_{s}, \theta_{*}=D_{\mathrm{OL}} \theta / r_{s}$, and then $\xi_{*}=\xi / r_{s}=\theta_{*}$. The latter equation means that the normalized variables $\xi_{*}$ and $\theta_{*}$ can be used interchangeably, and then hereafter we will use $\theta_{*}$ as our distance variable. ${ }^{5}$ Likewise, the total mass, as given in Equation 3(a), is normalized as

$$
m_{*}\left(\theta_{*}\right)=\frac{m\left(\theta_{*}\right)}{\rho_{\mathrm{s}} r_{\mathrm{s}}^{3}}=2 \pi \int_{0}^{\theta_{*}} d \hat{\theta}_{*} \hat{\theta}_{*} \Sigma_{*}\left(\hat{\theta}_{*}\right) .
$$

The normalized projected surface mass density, from Equation $3(\mathrm{~b})$, is

$$
\Sigma_{*}\left(\theta_{*}\right)=\frac{\Sigma\left(\theta_{*}\right)}{\rho_{\mathrm{s}} r_{\mathrm{s}}}=2 \int_{0}^{z_{\max *}} d z f\left(z, \theta_{*}\right),
$$

with $z=\sqrt{r_{*}^{2}-\theta_{*}^{2}}$ and $r_{*}=r / r_{s}$. The new parameter $\lambda$ in Equation (4) is then given by

$$
\lambda \equiv \frac{\rho_{\mathrm{s}} r_{\mathrm{s}}}{\pi \Sigma_{\mathrm{cr}}}=10^{-3} \frac{0.57}{h}\left(\frac{\rho_{\mathrm{s}} r_{s}}{M_{\odot} \mathrm{pc}^{-2}}\right) \frac{d_{\mathrm{OL}} d_{\mathrm{LS}}}{d_{\mathrm{OS}}},
$$

where we have defined the reduced (dimensionless) angular distances $d_{A}=D_{A} H_{0} / c$. The angular diameter distance $D_{A}$ as a function of redshift is computed in the standard way (see, e.g., Hogg 1999), assuming cosmological model parameters as given by the Planck 2015 results (Planck Collaboration et al. 2016). Equation (6) contains information about the lensing properties of any given model, together with that of the different distances involved in the lens system, namely, between the observer and the lens (OL), between the observer and the source (OS), and between the lens and the source (LS). ${ }^{6}$

One particular case of interest is that of perfect alignment between the luminous source and the lens system for which $\beta_{*}\left(\theta_{* E}\right)=0$. This in turn defines an Einstein ring with radius $R_{E}=D_{\mathrm{OL}} \theta_{E}$ and an associated angular radius $\theta_{E}$. In terms of our normalized variables, we see that the normalized angular Einstein radius $\theta_{* E}$ directly is the ratio of the Einstein radius to the (characteristic) scale radius of the density profile, $\theta_{* E}=R_{E} / r_{s}$. Moreover, the angular radius $\theta_{* E}$ must also be a solution of the equation (see Equation (4))

$$
\lambda=\frac{\theta_{* E}^{2}}{m_{*}\left(\theta_{* E}\right)} .
$$

Interestingly enough, Equation (7) shows that the lensing properties of a system with a density profile of the form $\rho(r)=\rho_{s} f\left(r / r_{s}\right)$ are independent of the density and distance scales and are mostly sensitive to the particular shape of the density profile. The physical parameters of the system are then concentrated in the dimensionless parameter $\lambda$ in Equation (6), and the latter can be calculated from Equation (7) without any prior knowledge of the given physical scales in the system, namely, $\rho_{s}$ and $r_{s}$, under only the assumption of perfect alignment (see Figure 1 for an example).

There is a critical value $\lambda_{\text {cr }}$ that is the smallest value of $\lambda$ for which an Einstein ring appears, which must correspond to the

\footnotetext{
5 For the sake of simplicity in the notation, we are using the same same angular variables (together with an asterisk) to denote the new normalized distances.

6 This is the same parameter used in González-Morales et al. (2013), but also see Park \& Ferguson (2003), in which the definition of $\lambda$ differs by a factor of $1 / 4 \pi$.
} 


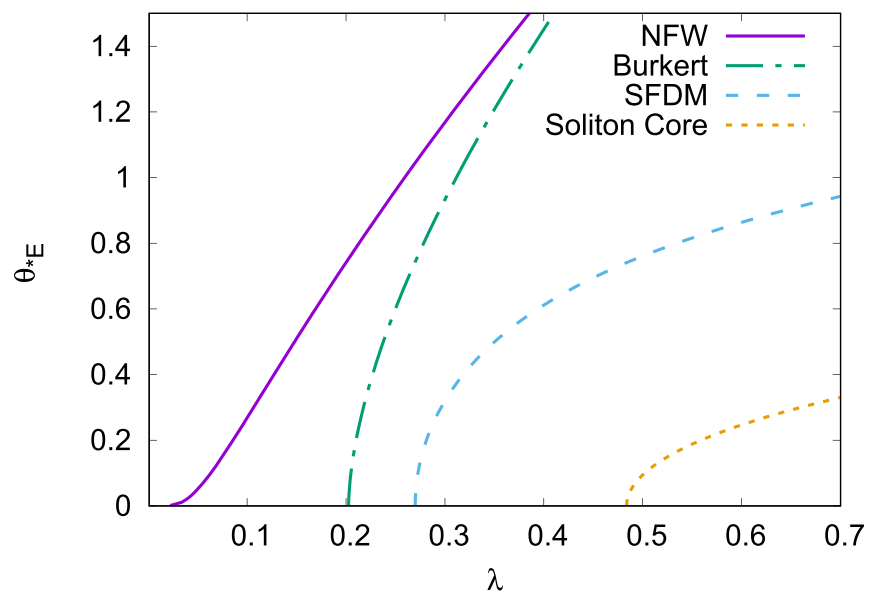

Figure 1. Normalized Einstein radius $\theta_{* E}$ as a function of $\lambda$ for different density profiles; see Equation (7). The point where each curve crosses the horizontal axis indicates the (intrinsic) critical value $\lambda_{\text {cr }}$ for each profile, in agreement with the values calculated from Equation (8) as shown in Table 1.

limit $\theta_{* E} \rightarrow 0$ in Equation (7). As we shall show now, such a critical value can be calculated analytically in the general case. To avoid the divergence at $\theta_{* E}=0$ (where $m_{*}(0)=0$ ), we make use of the L'Hôpital rule in Equation (7), and from Equation 3(a) we finally obtain

$$
\lambda_{\mathrm{cr}}^{-1}=\pi \Sigma_{*}(0)=2 \pi \int_{0}^{r_{\max *}} d \hat{r}_{*} f\left(\hat{r}_{*}\right),
$$

where $\Sigma(0)$ is the central value of the projected surface mass density given by Equation 3(b). Equation (8) is quite a simple formula for the calculation of $\lambda_{\mathrm{cr}}$ for any given density profile $\rho(r){ }^{7}$

As said before, Equation (8) suggests that the critical value $\lambda_{\text {cr }}$ just depends on the particular shape of the given density profile and no information is necessary about its other physical parameters. The values of $\lambda_{\text {crit }}$, calculated from Equation (8) for density profiles that are well known in the literature, are shown in Table 1. For these profiles we also show in Figure 1 the Einstein angle $\theta_{* E}$ as calculated from Equation (7). As expected, the Einstein angle is the smallest for the WaveDM profile (Equation (10)) alone, which also means that it is the one with the weakest lensing signal.

We should mention here an additional use of Equation (7): to constrain the free parameters of a given density profile. This relates to the fact that any DM halo characterized by a particular density profile needs to satisfy the constraint $\lambda \geqslant \lambda_{\mathrm{cr}}$ if it is to produce a lensing signal. Using Equations (6) and (8), the latter statement can be rewritten as

$$
\frac{\rho_{s} r_{s}}{M_{\odot} \mathrm{pc}^{-2}} \geqslant 10^{3} \frac{h}{0.57} \frac{d_{\mathrm{OS}}}{d_{\mathrm{OL}} d_{\mathrm{LS}}} \lambda_{\mathrm{cr}} .
$$

Equation (9) establishes a minimum value for the (structural) surface density $\rho_{s} r_{s}$ of any given DM profile in terms of the measured quantities of a lens system. Although the constraint Equation (9) is satisfied automatically by the NFW profile, for which $\lambda_{\text {crit }}=0$, this is not the case for the other profiles listed in Table 1 .

\footnotetext{
7 It should be noted that the definition of $\lambda_{\mathrm{cr}}$ depends on the chosen scale radius for normalization $r_{s}$, so that the value obtained from Equation (8) in our case is considering that $r_{s}$ coincides with the intrinsic distance scale in the density profile $\rho(r)$.
}

\subsection{Combined Density Profile of WaveDM}

For the density profile of WaveDM halos we will consider the model described in Schive et al. (2014a, 2014b), which arises from the study of extensive $N$-body simulations. The profile consists basically of two parts: one part describing a core sustained by the quantum pressure of the boson particles, also known as the soliton profile, and another part that resembles an NFW-like profile in the outer parts of the halo. As argued in Marsh \& Pop (2015), the transition at some radius to an NFW profile must be expected from the change of behavior to CDM on scales larger than the natural length of coherence, which should be proportional to the associated Compton length of the boson particles (in full units, the Compton length is $L_{C}=\hbar /(m c)$, where $\hbar$ is the (reduced) Planck's constant and $c$ is the speed of light).

The soliton profile is given by

$$
\rho_{\text {sol }}(r)=\frac{\rho_{s}}{\left(1+r^{2} / r_{s}^{2}\right)^{8}}
$$

where $r_{s}$ and $\rho_{s}$ are its characteristic radius and central density contrast, respectively. This profile was first studied in detail in Schive et al. (2014b), although here we are following the nomenclature adopted in Marsh \& Pop (2015), where it is also shown that the profile fits well the ground-state solution of the so-called SP system of equations (Ruffini \& Bonazzola 1969; Guzman \& Urena-Lopez 2004). In this respect, the soliton profile is strongly related to the wave properties (via the Schrödinger equation) of the boson particles.

One important property of the profile given in Equation (10) is that it must also obey the intrinsic scaling symmetry of the SP system (Guzman \& Urena-Lopez 2004). If $0<\hat{\lambda} \ll 1$ is a constant parameter, it can be shown that the central density and radius in the soliton profile are given by

$$
\rho_{s}=\hat{\lambda}^{4} m_{a}^{2} m_{\mathrm{Pl}}^{2} / 4 \pi, \quad r_{s}=\left(0.23 \hat{\lambda} m_{a}\right)^{-1} .
$$

This equation suggests that the intrinsic, physical quantities of the soliton profile in Equation (10) are related as shown in Equation (1). This relation will be important later when we discuss the constraints on the boson mass $m_{a}$.

For the NFW profile at the outskirts of the galaxy halo we adopt the following parameterization:

$$
\rho_{\mathrm{NFW}}(r)=\frac{\rho_{s} \rho_{\mathrm{NFW} *}}{\alpha_{\mathrm{NFW}}\left(r / r_{s}\right)\left(1+\alpha_{\mathrm{NFW}} r / r_{s}\right)^{2}} .
$$

Notice that in writing Equation (12) we are assuming the following implicit definitions for the scale radius and density, respectively, of the NFW profile: $r_{\mathrm{NFW}}=r_{s} / \alpha_{\mathrm{NFW}}$ and $\rho_{\mathrm{NFW}}=\rho_{s} \rho_{\mathrm{NFW} *}$, where both $\alpha_{\mathrm{NFW}}$ and $\rho_{\mathrm{NFW} *}$ are dimensionless numbers.

Unfortunately, there is not precise information in Schive et al. (2014a) about the transition in a galaxy halo from the soliton profile of Equation (10) to the NFW profile of Equation (12) in the general case. Hence, for the present work we adopt the convention for a combined profile as suggested in Marsh \& Pop (2015),

$$
\rho(r)=\Theta\left(r_{\epsilon}-r\right) \rho_{\mathrm{sol}}(r)+\Theta\left(r-r_{\epsilon}\right) \rho_{\mathrm{NFW}}(r) .
$$

where $\Theta\left(r_{\epsilon}-r\right)$ is the Heaviside step function. Here $r_{\epsilon}$ is the matching radius where the transition between the individual profiles occurs, and which satisfies the condition $\rho\left(r_{\epsilon}\right)=\epsilon \rho_{s}$. 

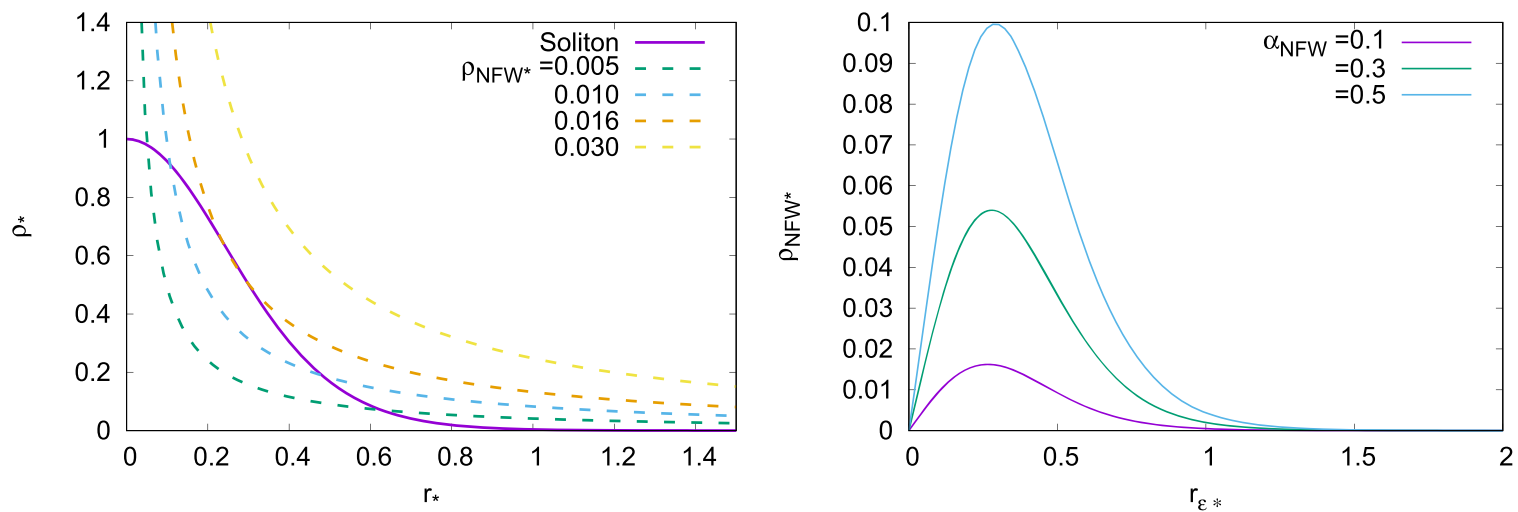

Figure 2. Determination of the matching radius, $r_{\epsilon *}$, for the density profile in Equation (14). Left: normalized soliton and NFW density profiles showing that there are at most two possible for $r_{\epsilon *}$ for each combination of $\alpha_{\mathrm{NFW}}$ (set to 0.1 only for illustrative purposes) and $\rho_{\mathrm{NFW} *}$. Right: NFW normalizing density factor $\rho_{\mathrm{NFW} *}$ as a function of $r_{\epsilon *}$ for different values of $\alpha_{\mathrm{NFW}}$, as obtained from Equation 15(c).

Table 1

The Intrinsic Value $\lambda_{\text {cr }}$, Calculated from Equation (8) for Halos with Different Density Profiles

\begin{tabular}{llcl}
\hline \hline Name & Density Profile $f(r)$ & $\lambda_{\text {cr }}$ & References \\
\hline NFW & {$\left[\left(r / r_{s}\right)\left(1+r / r_{s}\right)^{2}\right]^{-1}$} & 0 & Wright \& Brainerd (2000) \\
Burkert & {$\left[\left(1+r / r_{s}\right)\left(1+r / r_{s}^{2}\right)\right]$} & $2 / \pi^{2} \simeq 0.203$ & Park \& Ferguson (2003) \\
SFDM & $\sin \left(\pi r / r_{s}\right) /\left(\pi r / r_{s}\right)$ & 0.27 & González-Morales et al. (2013) \\
WaveDM & $\left(1+r^{2} / r_{s}^{2}\right)^{-8}$ & $\frac{2048}{429 \pi^{2}} \simeq 0.484$ & Schive et al. (2014b), Marsh \& Pop (2015) \\
\hline
\end{tabular}

Notice that $0<\epsilon<1$ if the transition between the profiles is to occur at the outskirts of the galaxy halo.

In general terms, and under our parameterization, there are six free parameters in the combined profile (Equation (13)): $\left(\rho_{s}, r_{s}, \rho_{\mathrm{NFW} *}, \epsilon, r_{\epsilon}, \alpha_{\mathrm{NFW}}\right)$. We will now derive two new constraints that arise from the continuity of the combined density profile at the matching radius, which will help us to reduce the number of free parameters.

For a continuous density function, we must impose the condition

$$
\rho_{\mathrm{sol}}\left(r_{\epsilon}\right)=\epsilon \rho_{s}=\rho_{\mathrm{NFW}}\left(r_{\epsilon}\right) .
$$

When Equation (14) is applied to the soliton profile of Equation (10), we obtain

$$
r_{\epsilon *}=r_{\epsilon} / r_{s}=\left(\epsilon^{-1 / 8}-1\right)^{1 / 2},
$$

which basically establishes the interchangeability of the (dimensionless) matching radius $r_{\epsilon *}$ and $\epsilon$. In the case of the NFW profile (Equation (12)), the continuity condition (Equation (14)) establishes that

$$
\epsilon^{-1} \rho_{\mathrm{NFW} *}=\alpha_{\mathrm{NFW}} r_{\epsilon *}\left(1+\alpha_{\mathrm{NFW}} r_{\epsilon *}\right)^{2},
$$

which, taking into account Equation 15(a), can be written as

$$
\rho_{\mathrm{NFW} *}=\frac{\alpha_{\mathrm{NFW}} r_{\epsilon *}\left(1+\alpha_{\mathrm{NFW}} r_{\epsilon *}\right)^{2}}{\left(1+r_{\epsilon *}^{2}\right)^{8}} .
$$

Equation 15(c) indicates the (normalized) density $\rho_{\mathrm{NFW} *}$ that is required for a correct matching between the soliton and NFW profiles, for given values of $\alpha_{\mathrm{NFW}}$ and $r_{\epsilon *}$.

However, one can see that the continuity constraint (Equation 15(c)) actually shows a hidden degeneracy: once the values of $\alpha_{\mathrm{NFW}}$ and $\rho_{\mathrm{NFW} *}$ are fixed, there can be up to two solutions for the matching radius $r_{\epsilon *}$. This is a direct consequence of the fact that the crossing of the density profiles
(Equations (10) and (12)) can occur at most at two different points, as illustrated in the left panel of Figure 2, which shows normalized density profiles for $\alpha_{\mathrm{NFW}}=0.1$ and different values of the normalized density $\rho_{\mathrm{NFW} *}$.

Figure 2 (left panel) also shows that there exists a maximum value of $\rho_{\mathrm{NFW} *}$ beyond which the profiles do not cross each other. This fact can be understood in terms of Equation 15(c), which we evaluate for different values of $\alpha_{\mathrm{NFW}}$ in the right panel of Figure 2. Here we can see that, for each $\alpha_{\mathrm{NFW}}$, there is always a maximum value of $\rho_{\mathrm{NFW} *}$, as a function of the matching radius $r_{\epsilon *}$, that corresponds to the case in which the soliton and NFW density profiles barely touch, as seen in the left panel of Figure 2.

To avoid the hidden degeneracy, and to select always a combined profile with an interior soliton shape, we will choose those cases for which $r_{\epsilon *} \geqslant r_{\epsilon *, \max }$, where $r_{\epsilon * \text { max }}$ is the matching radius corresponding to the maximum value of $\rho_{\mathrm{NFW} *}$. A straightforward calculation from Equation 15(c) shows that $r_{\epsilon * \text { max }}$ is a root of the cubic equation

$$
13 \alpha_{\mathrm{NFW}} r_{\epsilon *, \text { max }}^{3}+15 r_{\epsilon *, \text { max }}^{2}-3 \alpha_{\mathrm{NFW}} r_{\epsilon *, \max }=1 .
$$

Although there is a general solution to this equation, it can be shown that the limits for small and large values of $\alpha_{\mathrm{NFW}}$ are

$$
\begin{aligned}
\lim _{\alpha_{\mathrm{NFW}} \rightarrow 0} r_{\epsilon *, \max } & =(1 / \sqrt{15}), \\
\lim _{\alpha_{\mathrm{NFW}} \rightarrow \infty} r_{\epsilon *, \max } & =(\sqrt{3 / 13}) .
\end{aligned}
$$

This means that in absolute terms the maximum value of $\rho_{\mathrm{NFW} *}$ must be located in the range $0.25<r_{\epsilon * \text { max }}<0.48$, which is in agreement with the values observed in the right panel of Figure 2. After all this, it is possible to reduce the number of free parameters that describe the combined profile (13) to only four: $\rho_{s}, r_{s}, r_{\epsilon}$, and 
$\alpha_{\mathrm{NFW}}$. By means of these parameters and the constraints discussed above, the other parameters are fully specified.

Notice that our chosen normalization is such that the physical parameters in the NFW profile (Equation (12)) are given in terms of those in the soliton profile (Equation (10)). This means, for instance, that $\rho_{\mathrm{NFW} *}>1\left(\rho_{\mathrm{NFW} *}<1\right)$ is equivalent to $\rho_{\mathrm{NFW}}>\rho_{s}\left(\rho_{\mathrm{NFW}}<\rho_{s}\right)$, whatever the physical value of $\rho_{s}$ is. Likewise, we find that $\alpha_{\mathrm{NFW}}<1\left(\alpha_{\mathrm{NFW}}>1\right)$ corresponds to $r_{\mathrm{NFW}}>r_{s}\left(r_{\mathrm{NFW}}<r_{s}\right)$, even if the physical value of $r_{s}$ is not known beforehand. The same will apply for the matching radius, since $r_{\epsilon *}>1 \quad\left(r_{\epsilon *}<1\right)$ means that matching occurs beyond the soliton radius and then $r_{\epsilon}>r_{s}$ (before the soliton radius and then $r_{\epsilon}<r_{s}$ ).

It must be noticed also that the prescription above for the matching of the density profiles in Equation (13) means that the NFW part is always subjected to the presence of the central soliton. For instance, the NFW profile can be diluted away if the matching radius $r_{\epsilon *} \rightarrow \infty$ (which also means that $\rho_{\mathrm{NFW} *} \rightarrow 0$ ), and then the density profile becomes the soliton one alone, $\rho(r) \simeq$ $\rho_{\text {sol }}(r)$. On the other hand, if $r_{s} \rightarrow 0$, the central soliton becomes small but much more massive and denser, because of the scaling symmetry shown in Equation (1), so that it dominates the matter contents over that of the NFW profile. The conclusion here is that under our parameterization the density profile (Equation (13)) can become the soliton profile only if $r_{\epsilon *} \rightarrow \infty$, but it is not possible to do the same for the NFW part; in this sense, the complete profile (Equation (13)) should always be seen as that of a central soliton with a subdominant NFW tail.

We want to stress that the complete profile (13) should not be confused with the so-called cored NFW profile that exists already in the literature. The latter is of the form $\rho(r) \sim\left(r / r_{s}\right)^{-\beta} /\left(1+r / r_{s}\right)^{2}$ with $0<\beta<1$, and whose lensing properties have been analyzed in Wyithe et al. (2001). A comparison of the lenses produced by the cored NFW profile and the WaveDM one is beyond the purpose of this work, as our primary intention is to constrain the parameters in Equation (13) and to obtain from them credible bounds on the mass of the boson particles.

As a final note, we emphasize the convenience of the chosen parameterization in terms of the soliton characteristic quantities, as the soliton and NFW parameters must follow well-defined scaling constraints that are intrinsic to the WaveDM. These scaling properties will then be already explicit in the complete profile (13) when making a comparison of the model with lensing data.

\subsection{Gravitational Lensing}

To obtain the lensing properties of the combined profile given by Equation (13), we follow the recipe described in Section 2.1. We first need to compute the projected surface mass density (Equation 3(b)). Because of the presence of the step functions in Equation (13), the integral in Equation 3(b) naturally separates as
It should be understood that the integrals in Equation (18) are done along the line of sight. Notice also that we are following our convention in Section 2 for normalized quantities, namely, $\Sigma_{*}=\Sigma /\left(\rho_{s} r_{s}\right), \theta_{*}=\xi / r_{s}$ and $z=\sqrt{r_{*}^{2}-\theta_{*}^{2}}$. The analytical expressions for the integrals in Equation (18) can be found in the Appendix.

Equation (18) shows that the projected surface mass density only depends on the characteristic radii and densities of the combined density profile (Equation (13)). For instance, if we keep $r_{s}$ fixed, it can be shown that

$$
\lim _{r_{\epsilon *} \rightarrow \infty} \Sigma_{*}\left(\theta_{*}, r_{\epsilon *}, \alpha_{\mathrm{NFW}}\right)=0.658\left(1+\theta_{*}^{2}\right)^{-15 / 2}
$$

a result that is obtained from the first branch in Equation (18). Notice that Equation (19) is exactly the result for the soliton profile (Equation (10)) alone. Also, as we have mentioned before, it is not possible to recover the standard result of the surface density for the NFW profile by letting $r_{s} \rightarrow 0$, as in this case the matter content is still dominated by the central soliton.

Going back to the complete profile (Equation (13)), we start with the calculation of the critical value $\lambda_{\text {crit }}$ from the analytical formula in Equation (8). The (total) projected surface mass density for the special value $\theta_{*}=0$ is obtained from the first branch, in Equation (18), as

$$
\begin{aligned}
& \Sigma_{*}\left(0, r_{\epsilon *}, \alpha_{\mathrm{NFW}}\right) \\
& =2\left[\int_{0}^{r_{\epsilon *}} \frac{d z}{\left(1+z^{2}\right)^{8}}+\frac{r_{\epsilon *}\left(1+\alpha_{\mathrm{NFW}} r_{\epsilon *}\right)^{2}}{\left(1+r_{\epsilon *}^{2}\right)^{8}} \int_{r_{\epsilon *}}^{\infty} \frac{d z}{z\left(1+\alpha_{\mathrm{NFW}} z\right)^{2}}\right],
\end{aligned}
$$

which indicates, together with Equation (7), that the critical value $\lambda_{\text {cr }}$ of the combined profile (Equation (13)) is a function of $r_{\epsilon *}$ and $\alpha_{\mathrm{NFW}}$, and its behavior for different combinations of these parameters is shown in the left panel of Figure 3. Notice that we have taken into account the constraint $r_{\epsilon *} \geqslant r_{\epsilon * \text {,max }}$; see Equation (16). Moreover, it can be seen that the lowest value of $\lambda_{\mathrm{cr}}$, for any given value of $\alpha_{\mathrm{NFW}}$, is indeed attained at $r_{\epsilon * \text {,max }}$ as indicated by the vertical lines with the corresponding colors. Not surprisingly, the addition of the NFW outer part helps the soliton profile to achieve small values of $\lambda_{\text {crit }}$, which in turn eases the accomplishment of the inequality in Equation (9). In particular, Figure 3 shows that $\lambda_{\text {crit }} \rightarrow 0$ as $\alpha_{\mathrm{NFW}} \rightarrow 0$, which means that the combined profile (13) will be able to produce a lensing signal for any nontrivial combination of its parameters $\rho_{s}$ and $r_{s}$.

In the case of the combined profile the total mass $M(r)$ inside a sphere of any given radius $r>r_{\epsilon}$ is simply given by

$$
\Sigma_{*}\left(\theta_{*}, r_{\epsilon *}, \alpha_{\mathrm{NFW}}\right)=2 \begin{cases}\int_{0}^{\sqrt{r_{\epsilon *}^{2}-\theta_{*}^{2}}} \frac{d z}{\left(1+\hat{r}^{2}\right)^{8}}+\frac{r_{\epsilon *}\left(1+\alpha_{\mathrm{NFW}} r_{\epsilon *}\right)^{2}}{\left(1+r_{\epsilon *}^{2}\right)^{8}} \int_{\sqrt{r_{\epsilon *}^{2}-\theta_{*}^{2}} \frac{d z}{\hat{r}\left(1+\alpha_{\mathrm{NFW}} \hat{r}\right)^{2}},}^{\infty} \theta_{*}<r_{\epsilon *}, \\ \frac{r_{\epsilon *}\left(1+\alpha_{\mathrm{NFW}} r_{\epsilon *}\right)^{2}}{\left(1+r_{\epsilon *}^{2}\right)^{8}} \int_{0}^{\infty} \frac{d z}{\hat{r}\left(1+\alpha_{\mathrm{NFW}} \hat{r}\right)^{2}}, & \theta_{*} \geqslant r_{\epsilon *}\end{cases}
$$



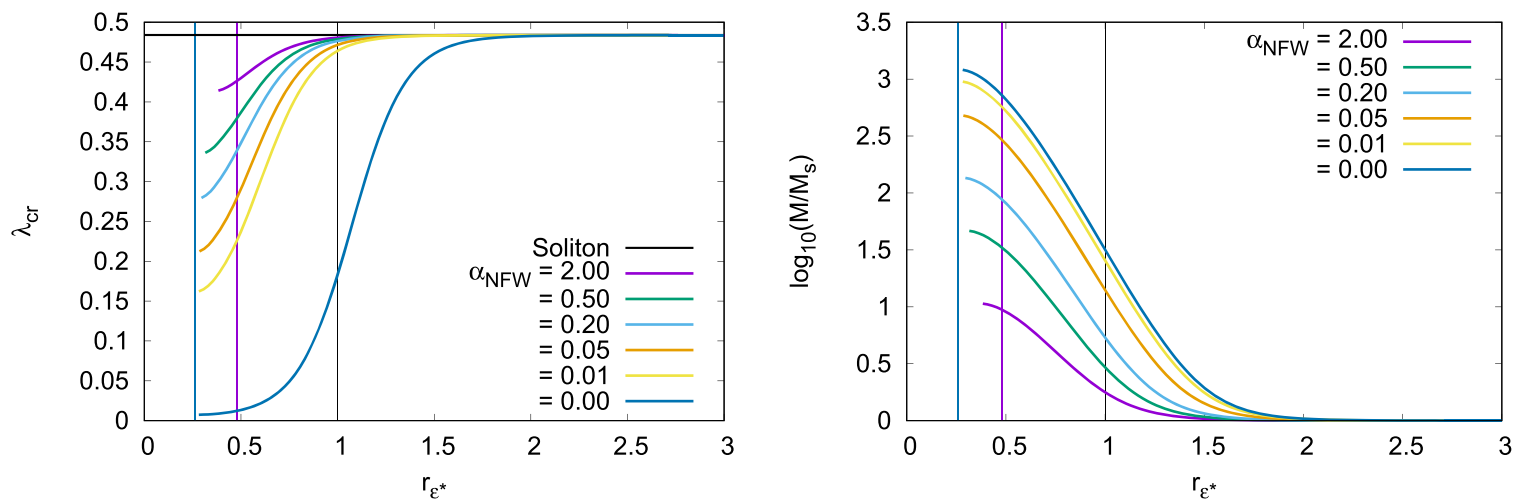

Figure 3. Critical value $\lambda_{\text {cr }}$ to produce strong lensing (left) and total lens mass $M$ normalized by the soliton mass $M_{s}$ (right), both as a function of the matching radius $r_{\epsilon *}$ for different density profiles characterized by $\alpha_{\mathrm{NFW}}$. The pure soliton case, $\lambda_{\mathrm{cr}} \simeq 0.48$, is recovered asymptotically in the limit $r_{\epsilon *} \rightarrow \infty$. Vertical lines mark the position of the normalized soliton radius at $r_{\epsilon *}=1,0.5,0.25$ (gray, purple, and blue, respectively). The presence of the NFW part eases the formation of multiple images. See the text for more details.

the integral

$$
\begin{aligned}
& \frac{M(r)}{10^{13} M_{\odot}}=3\left(\frac{m_{a}}{10^{-22} \mathrm{eV}}\right)^{-2}\left(\frac{r_{s}}{\mathrm{pc}}\right)^{-1} \\
& \times\left[\int_{0}^{r_{\epsilon *}} \frac{d x x^{2}}{\left(1+x^{2}\right)^{8}}+\frac{r_{\epsilon *}\left(1+\alpha_{\mathrm{NFW}} r_{\epsilon *}\right)^{2}}{\left(1+r_{\epsilon *}^{2}\right)^{8}} \int_{r_{\epsilon *}}^{r_{*}} \frac{d x x}{\left(1+\alpha_{\mathrm{NFW}} x\right)^{2}}\right] .
\end{aligned}
$$

In the general case the total mass diverges as $r \rightarrow \infty$, whereas for the soliton profile only (which requires $r_{\epsilon *} \rightarrow \infty$ ) we simply obtain that its total mass $M_{s}$ is (Guzman \& Urena-Lopez 2004; Marsh \& Pop 2015; Chen et al. 2017)

$$
\frac{M_{s}}{10^{11} M_{\odot}}=7.7\left(\frac{m_{a}}{10^{-22} \mathrm{eV}}\right)^{-2}\left(\frac{r_{s}}{\mathrm{pc}}\right)^{-1} .
$$

In general, we expect from Equation (20) the total mass in the combined profile to be larger than the soliton alone, that is, $M(r) \geqslant M_{s}$. However, the value of the total mass $M$ will depend on the upper limit of integration $r_{*}$, and the largest values for any given $r_{*}$ will be obtained for the case where $\alpha_{\mathrm{NFW}} \rightarrow 0$, similar to the case of the critical value $\lambda_{\text {crit }}$. The aforementioned general behavior of the total mass $M$ as a function of the free parameters $r_{\epsilon *}$ and $\alpha_{\mathrm{NFW}}$ is shown in the right panel of Figure 3. For the numerical examples we considered the upper limit of integration $r_{*}=20$, for which we then see that that the difference between $M$ and $M_{s}$ can be as large as three orders of magnitude in the case $\alpha_{\mathrm{NFW}}=0$. In other words, the total mass in the WaveDM profile is intrinsically attached to that of its soliton, and then the latter should be large enough if we are going to get the right mass scales in galaxies. This is a nontrivial property, as it shows that any nonzero value of the parameter $\alpha_{\mathrm{NFW}}$ could point out the existence of a soliton core with a non-negligible mass contribution to the lens system (see, e.g., Figure 5 below).

\section{Data Analysis}

In this section we will use our theoretical results to infer information about the WaveDM profile from observations of specific lens systems. We recall from Section 2.2 that there are four free parameters that are needed to describe the lensing properties of the combined density profile, Equation (13). However, the lens equation, discussed in Section 2.3, is not explicitly dependent on two of them, namely, $\rho_{s}$ and $r_{s}$, and depends only on the free parameters of the NFW outer profile $r_{\epsilon *}$ and $\alpha_{\mathrm{NFW}}$. Therefore, we could use the right-hand side of the lens equation (Equation (4)) to put constraints on the surface density through the combination of parameters $\rho_{s} r_{s}$; see also the discussion in Section 2.1.

However, the special properties of the WaveDM profile, as represented by Equation (1), suggest that the lens equation could be written in a more convenient form. Using the fact that the (normalized) angular Einstein radius is $\theta_{* E}=R_{E} / r_{s}$, Equation (6) can be recast in the form

$$
m_{a 22}^{-2} \theta_{* E} m_{*}\left(\theta_{* E}, \alpha_{\mathrm{NFW}}, r_{\epsilon *}\right)=\frac{1}{2.4} \frac{d_{\mathrm{OS}}}{d_{\mathrm{OL}} d_{\mathrm{LS}}} \frac{h}{0.57}\left(\frac{R_{E}}{\mathrm{kpc}}\right)^{3},
$$

where we have set $m_{a 22} \equiv m_{a} / 10^{-22} \mathrm{eV}$. Equation (22) then defines a different observable, which results solely from the combination of the distances involved in the measurement of the lens system, so that we can put constraints directly on the boson mass $m_{a}$ rather than on the energy density $\rho_{s}$, but in any case in combination with the rest of the parameters, namely, $\theta_{* E}, \alpha_{\mathrm{NFW}}$, and $r_{\epsilon *}$.

In general, we expect that, given the data from a single galaxy, there will always be a region in the parameter space that will satisfy Equation (22). Thus, for a given sample of galaxies, we could in principle determine the range of possible values of $m_{a}$ that is consistent with the observed data. However, we must recall that the boson mass $m_{a}$ is a fundamental physical parameter of the model that in principle should have a unique value. This means that the boson mass should be treated differently from other parameters in the model and should not be given the freedom to vary from galaxy to galaxy.

Our proposal, therefore, is to study the lensing properties of the WaveDM profile by fixing the value of the boson mass and finding, via statistical analysis, the best-fit values of the remaining free parameters $\theta_{* E}, \alpha_{\mathrm{NFW}}$, and $r_{\epsilon *}$. As we are interested in the properties of the WaveDM profile alone, we select a particular subsample of early-type galaxies, and we will focus in particular on those lensed systems in which the galaxy is known to have a relatively high DM fraction. We have set a threshold at a fraction of luminous matter of $50 \%$ or less, i.e., reducing as much as possible its effects. Also, due to the 
Table 2

Selected Galaxies from SLACS, LSD, and SL2S

\begin{tabular}{lcllrl}
\hline \hline Name & $f_{*, \text { Ein }}^{\text {Salp }}$ & $z_{\text {lens }}$ & $z_{\text {source }}$ & $d_{\mathrm{OS}} /\left(d_{\mathrm{OL}} d_{\mathrm{LS}}\right)$ & $R_{E}(\mathrm{kpc})$ \\
SLACS & & & & & \\
\hline J0008-0004 & $0.50 \pm 0.16$ & 0.44 & 1.192 & 6.6855 & 6.7965 \\
J0935-0003 & $0.35 \pm 0.05$ & 0.347 & 0.467 & 18.2172 & 4.4063 \\
J0946+1006 & $0.46 \pm 0.13$ & 0.222 & 0.609 & 9.7613 & 5.0934 \\
J1143-0144 & $0.46 \pm 0.10$ & 0.106 & 0.402 & 14.9617 & 3.3683 \\
J1306+0600 & $0.47 \pm 0.08$ & 0.173 & 0.472 & 11.7208 & 4.0050 \\
J1318-0313 & $0.42 \pm 0.08$ & 0.24 & 1.3 & 7.2634 & 6.1840 \\
LSD & & & & & \\
CFRS & $0.46 \pm 0.15$ & 0.94 & 2.94 & 5.3188 & 10.0470 \\
$\quad$ 03.1077 & & & & & \\
HST & $0.38 \pm 0.11$ & 0.81 & 3.40 & 4.7801 & 10.9360 \\
$\quad$ 1417 & & & & & \\
$\quad+5226$ & & & & & \\
SL2S & & & & & \\
J220329 & $0.24 \pm 0.06$ & 0.40 & 2.150 & 5.4526 & 10.8130 \\
$\quad+020518$ & & & & & \\
\hline
\end{tabular}

Note. Columns correspond to the following: label within the SDSS catalog (name), fraction of luminous matter within the Einstein radius $\left(f_{* \text { Ein }}^{\text {Salp }}\right)$, redshift of the lens $\left(z_{\text {lens }}\right)$ and the source $\left(z_{\text {source }}\right)$, distance factor $d_{\mathrm{OS}} /\left(d_{\mathrm{OL}} d_{\mathrm{LS}}\right)$, and measured Einstein radius $\left(R_{E}\right)$. Selection was based on the condition $f_{* \text { Ein }}^{\text {Salp }} \leqslant 0.5$.

consideration of the lens model to be composed of a single galaxy, samples where contribution of more than one component is known were excluded, e.g., MG $2016+112$ (see Nair \& Garrett 1996).

We use strongly lensed galactic-scale systems observed by the Sloan data from the Sloan Lens ACS (SLAC) survey, which is composed of nearly 100 likely and confirmed lensed systems (see Gavazzi et al. 2007; Auger et al. 2009). Our criteria reduce this sample to a subsample of only six galaxies. We also include samples from Lens Structure and Dynamics (LSD) and the Strong Lensing Legacy Survey (LS2S; see, e.g., Treu \& Koopmans 2004; Sonnenfeld et al. 2013a; Cao et al. 2015), but after applying the same criteria, we ended up selecting only three galaxies. The names of the nine chosen galaxies are shown in Table 2, together with the values of their lens parameters.

Another advantage from the SLAC survey is that it was analyzed previously with other, similar, scalar field DM models (see, e.g., González-Morales et al. 2013; Robles \& Matos 2013). Due to the lack of studies of lensing for this kind of model, we therefore adopt SLACS as the main subsample for consistency with previous studies and as a proof of concept for the possible use of the methodology.

The Einstein radius $R_{E}$ is obtained using $R_{E}=D_{\mathrm{OL}} \theta_{E}$, where $\theta_{E}$ is as given in the literature (see Treu \& Koopmans 2004; Bolton et al. 2008a; Auger et al. 2009; Sonnenfeld et al. $2013 \mathrm{~b})$. It has been shown that $\theta_{E}$ is nearly model independent and well constrained and has been used before to determine the lens mass where only big asymmetric arcs in the images could produce some difference ${ }^{8}$ (see, e.g., Kochanek 1991; Kochanek et al. 2001; Cardone et al. 2009; Trick et al. 2016; Lyskova et al. 2018; Tortora et al. 2018); this justifies its direct use as a reliable observable in our analysis. The distances and

\footnotetext{
8 In particular for SLACS, it is important to remark that they adjusted different mass models and found that the images were visually indistinguishable and the Einstein angles were the same within errors. This is stated in Section 5.2 and Table 5 of Bolton et al. (2008a).
}

$R_{E}$ values in Table 2 are obtained considering a cosmology with matter density parameter $\Omega_{M}=0.3089$, vacuum energy density parameter $\Omega_{\Lambda}=0.6911$, and Hubble parameter $H_{0}=$ $67.74 \mathrm{~km} \mathrm{~s}^{-1} \mathrm{Mpc}^{-1}$ from the Planck Collaboration et al. (2015).

\subsection{Soliton Core Profile}

As a first case of study, let us consider the soliton core profile without the external NFW part. There are in this case only two free parameters: $m_{a 22}$ and $\theta_{* E}$. In Section 3.2 a Bayesian analysis will be carried out, taking into account the results from this section. The projected mass surface density given by Equation 5(a), with the help of Equation (19), has in this case an analytical expression,

$$
m_{*}\left(\theta_{* E}\right)=\frac{2}{13 \lambda_{\text {crit }}} \frac{\left(1+\theta_{* E}^{2}\right)^{13 / 2}-1}{\left(1+\theta_{* E}^{2}\right)^{13 / 2}},
$$

where $\lambda_{\text {crit }} \simeq 0.484$ is the critical value calculated from Equation (8); see also Table 1 . Notice that $m_{*}(0)=0$, whereas its asymptotic limit is $m_{*}(\infty)=2 /\left(13 \lambda_{\text {crit }}\right)$.

To obtain a basic understanding of the solutions that will be found for the physical parameters, we show in the left panel of Figure 4 the expected behavior of the left-hand side of Equation (22) as a function of the Einstein angle $\theta_{* E}$. We also show, as the series of horizontal lines, the values of the righthand side of Equation (22) obtained from the observed data for the galaxies listed in Table 2 .

Figure 4 shows that it will always be possible to identify a value of the Einstein angle $\theta_{* E}$ for which the left-hand and right-hand sides of Equation (22) are in agreement, irrespective of the value of the boson mass-although as the boson mass increases, the agreement occurs at increasingly large values of $\theta_{* E}$. For the examples shown in Figure 4, a boson mass of order $m_{a 22} \simeq 0.02$ seems to fit well the SLACS galaxies listed in Table 2-corresponding to an allowed range for the angular Einstein radius of $5<\theta_{* E}<10$. The latter range can also be translated into an allowed range for the soliton radius and suggests that $r_{s} \sim \mathrm{kpc}$ for the given example galaxies. However, note that it is always possible to find a solution that matches the left-hand and right-hand sides of Equation (22), for any given value of the boson mass $m_{a}$, by a suitably large choice of Einstein angle $\theta_{* E}$, that is, by choosing $r_{s} \rightarrow 0$. We must recall that the latter condition means that the density profile is dominated by a very massive and compact soliton, but this can be in disagreement with other indications about the actual size of the DM halo in the lens galaxies.

To summarize, given that we have only one observable constraint, the most we can do is first to fix the value of the boson mass $m_{a}$ and from this to obtain constraints on the remaining free parameters that are consistent with that boson mass. Specifically, by adopting a proposed value for the boson mass $m_{a}$ in Equations (22) and (23), we can obtain for each galaxy the corresponding best-fit value for $\theta_{* E}$, and from that the best-fit value for $r_{s}$.

The results obtained for our selected sample of galaxies are shown in Table 3 and also plotted in the right panel of Figure 4. The latter figure speaks for itself and shows that the data points for all galaxies lie along the line with a constant soliton mass $M_{s} \simeq 10^{11} M_{\odot}$ (see Equation (21)), and (as required) all lie below the line that represents the inequality, Equation (9), for 

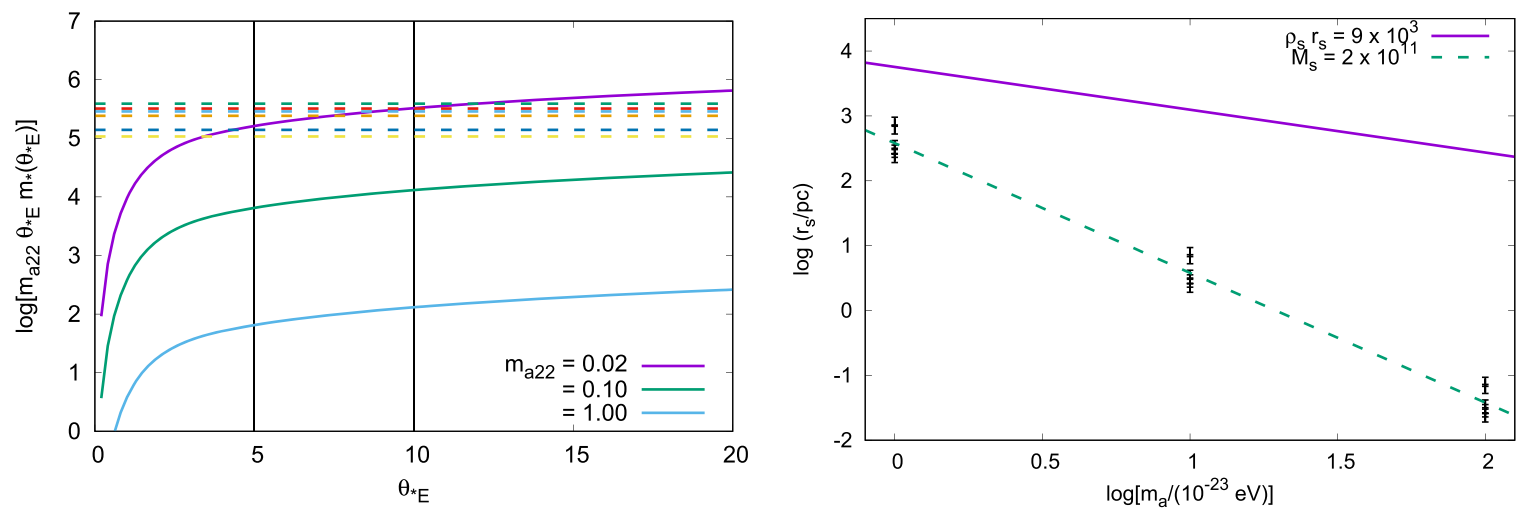

Figure 4. Left: left-hand side of Equation (22) as a function of the normalized Einstein angle, $\theta_{* E}=R_{E} / r_{s}$, according to Equation (23). The intersection with the dashed horizontal lines (right-hand side of Equation (22)) for each galaxy in our sample defines the value of $\theta_{* E}$, i.e the scale radius. Right: fitted soliton radius, $r_{s}$, as a function of the boson mass $m_{a}$. The resultant $r_{s}$ lies along the line of constant soliton mass $M_{s}$. This example is for $M_{s} \simeq 2 \times 10^{11} M_{\odot}$. See the text for more details.

Table 3

Soliton Radius, $r_{s}$, Obtained from the Fits to Each Galaxy and for Three Different Values of the Boson Mass $m_{a}$

\begin{tabular}{lccc}
\hline \hline & $m_{a 22}=10$ & $\begin{array}{c}m_{a 22}=1 \\
\log _{10}\left(r_{s} / \mathrm{pc}\right)\end{array}$ & $m_{a 22}=0.1$ \\
\cline { 2 - 4 } Galaxy & & & \\
\hline JLACS & $-1.67_{-0.06}^{+0.07}$ & $0.33_{-0.06}^{+0.07}$ & $2.33_{-0.06}^{+0.07}$ \\
J0008-0004-0003 & $-1.73_{-0.06}^{+0.07}$ & $0.27_{-0.06}^{+0.07}$ & $2.27_{-0.06}^{+0.07}$ \\
J0946+1006 & $-1.59_{-0.06}^{+0.07}$ & $0.41_{-0.06}^{+0.07}$ & $2.41_{-0.06}^{+0.07}$ \\
J1143-0144 & $-1.41_{-0.06}^{+0.07}$ & $0.59_{-0.06}^{+0.07}$ & $2.59_{-0.06}^{+0.07}$ \\
J1306+0600 & $-1.46_{-0.06}^{+0.07}$ & $0.54_{-0.06}^{+0.07}$ & $2.54_{-0.06}^{+0.07}$ \\
J1318-0313 & $-1.62_{-0.06}^{+0.07}$ & $0.37_{-0.06}^{+0.07}$ & $2.37_{-0.06}^{+0.07}$ \\
LSD & $-1.58_{-0.12}^{+0.17}$ & $0.42_{-0.12}^{+0.17}$ & $2.42_{-0.12}^{+0.17}$ \\
CFRS 03.1077 & $-1.7_{-0.12}^{+0.18}$ & $0.30_{-0.12}^{+0.18}$ & $2.30_{-0.12}^{+0.17}$ \\
HST 1417+5226 & $-1.90_{-0.05}^{+0.06}$ & $0.10_{-0.05}^{+0.06}$ & $2.10_{-0.05}^{+0.06}$ \\
SL2S & & & \\
J220329+020518 & & &
\end{tabular}

Note. Note that for SLACS samples, all combinations have a total soliton mass mass contained within the Einstein radius of $M_{s} \simeq 10^{11.5} M_{\odot}$. The LSD and SL2S samples have a total mass of $M_{s} \simeq 10^{11.8} M_{\odot}$.

the galaxy in Table 2 (J0935-0003) with the most extreme value for the ratio of distances on the right-hand side of Equation (22). The different values obtained for the characteristic radius $r_{s}$ give an enclosed mass that corresponds closely to the values reported in Auger et al. (2009). Nevertheless, these models are found to be considerably too compact when the characteristic radius and corresponding enclosed mass are considered together. For example, galaxy J0008-0004 has a value for $M_{\text {Eins }}=3.1 \times 10^{11} M_{\odot}$ that is comparable to the value of $M_{s}=3.4 \times 10^{11} M_{\odot}$ obtained using the best-fit parameters of the soliton model. Notwithstanding that the soliton model gives an enclosed mass that is adequate and realistic, we think that the characteristic radius is most definitely not so. This is by taking into consideration the results from rotation curves where the effects of DM are expected to be at larger radii than the luminous part of the galaxy, and this contrasts with the values obtained for the soliton alone where the mean effective radius for galaxy J0008-0004 is observed to be $r_{e} \approx 9.6 \mathrm{kpc}$, which is several orders of magnitude larger than the characteristic radius $r_{s}$ obtained for any of the different boson masses presented in Table 3, including the samples from the other surveys. Therefore, we think that the soliton profile alone is actually not helping to explain the distribution of DM around the selected galaxies in a consistent way.

There are two valuable lessons from the above exercise. The first one is that the soliton core profile alone will always be able to fulfill the lensing constraints even without the consideration of the NFW contribution given the Einstein radius as the only measurement to satisfy. This is not surprising, as the lensing equations can be solved even if we consider a point particle with the required total mass (which formally corresponds to the soliton core profile with $m_{a} \rightarrow \infty$ ). The second lesson is that even though the soliton profile may be adequate, formally speaking, to explain the lensing properties of the galaxies in Table 2, we will, in any case, have to consider the NFW outskirts in the complete profile (Equation (13)) in order to satisfy other constraints that suggest that the boson mass should be in the range $m_{a 22}=1-10$ (see Hui et al. 2017).

\subsection{Complete Profile}

Taking into account the above experience gained with the soliton profile alone, we will now consider the following procedure for the complete WaveDM profile. Since the total mass inside the Einstein radius is the only constraint provided by the lens systems, we will fix the values of the boson mass $m_{a}$ and soliton mass $M_{s}$. This approach is considered due to the results from the soliton analysis, where the boson mass can satisfy different values for the Einstein radius, and other studies have found that $m_{a}$ needs to be in a certain range. For this, we take the following values of the boson mass $m_{a 22}=0.1,1,10$, and for the soliton mass $\log _{10}\left(M_{s} / M_{\odot}\right)=11.5,10.5,9.5,8.5,7.5$, from which we will calculate the values of $r_{s}$ by means of Equation (21), which allows us to avoid a possible overcompensation of the soliton mass.

We will adopt a uniform prior for the other parameters over the following ranges: $\alpha_{\mathrm{NFW}}=[0: 10]$ and $r_{\epsilon \star}=\left[r_{\epsilon \star, \max }: 10\right]$. Here $r_{\epsilon *, \text { max }}$ is found from the cubic Equation (16) for a given value of $\alpha_{\mathrm{NFW}}$, and the extreme values $\alpha_{\mathrm{NFW}}=10$ and $r_{\epsilon *}=10$ are suggested by Figures 3 and 4 .

We will obtain the values of $\theta_{* E}$ by sampling from a Gaussian distribution, using the relation

$$
\theta_{* E}(p)=\theta_{* E m}+\sigma \sqrt{2} \operatorname{erf}^{-1}(2 p-1), \quad p \in(0,1) .
$$

The value for $\theta_{* E m}=R_{E} / r_{s}$ is the mean of the distribution using the observed value for the Einstein radius, $\sigma=0.05^{*} \chi$ is the 

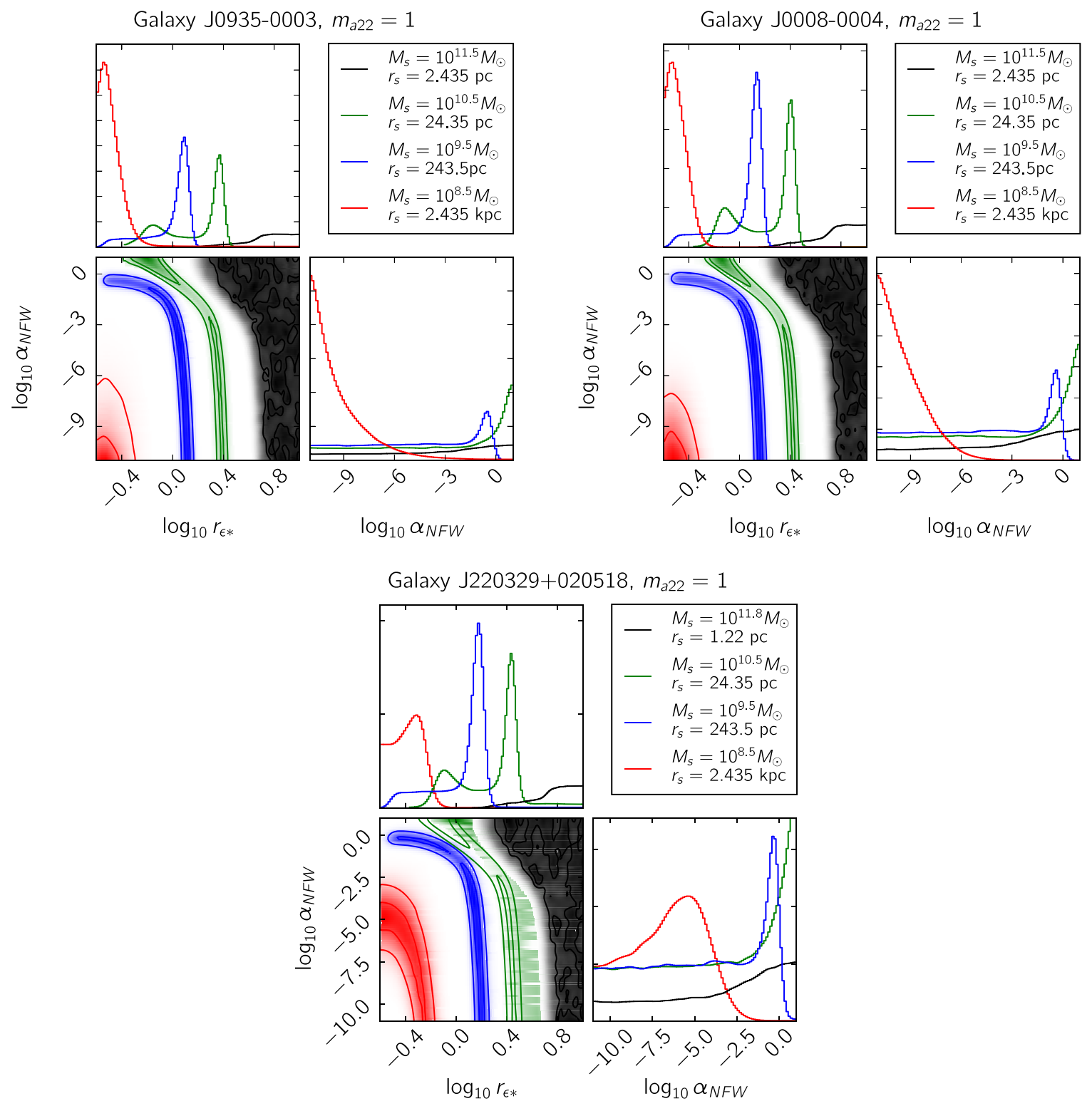

Figure 5. Posterior distribution for the parameters fitted to galaxies J0935-0003 (left), J0008-0004 (right), and J220329+020518 (bottom); the contribution of the luminous matter is $35 \%, 50 \%$, and $24 \%$, respectively, of the total reduced mass inside the Einstein radius. The colors indicate different combinations of the soliton mass, $M_{s}$, and scale radius, $r_{s}$, computed with a fixed (normalized) boson mass $m_{a 22}=1$.

error assigned, and $p$ is a random number sampled from a uniform distribution on the interval $[0,1]$. The inverse error function is approximated as described in Winitzki (2008). In this way, $\theta_{* E}$ will not enter into the fitting analysis as an extra variable.

Once the soliton mass is fixed, the rest of the mass that is included within the Einstein radius must be completed by the NFW profile. Because this requires a huge contribution, up to three orders of magnitude more, one sensible consideration is to set the total mass of the lens as composed by a simple representation of luminous matter and the selected model of DM. In a first approximation, the mass corresponding to the baryonic matter is simply a constant value modeled as a point particle. This is done from Equation (2), and then the projected mass for the lens is composed of two parts,

$$
m^{\prime}(\theta)=m(\theta)+M^{\prime}
$$

where $m(\theta)$ is the mass from the DM component given by the profile in Equation (13), and $M^{\prime}=f^{*}{ }_{\text {,Ein }} M_{\text {Ein }}$ is the stellar mass contribution as described in Table 2. These values are normalized accordingly, and then the dimensionless total mass $m^{\prime}$ is

$$
m_{*}^{\prime}\left(\theta_{* E}, \alpha_{\mathrm{NFW}}, r_{\epsilon *}\right)=m_{*}\left(\theta_{* E}, \alpha_{\mathrm{NFW}}, r_{\epsilon *}\right)+M_{*}^{\prime}
$$

where

$$
M_{*}^{\prime}=0.3208 f_{* \text { Ein }}\left(\frac{M_{\text {Ein }}}{M_{s}}\right)
$$

Equation 26(a) is combined with Equation (22) to produce a modified observable that uses the soliton mass directly,

$$
\frac{M_{s}}{M_{\odot}} m_{*}^{\prime}\left(\theta_{* E}, \alpha_{\mathrm{NFW}}, r_{\epsilon *}\right)=\frac{7.7 \times 10^{8}}{2.4} \frac{d_{\mathrm{OS}}}{d_{\mathrm{OL}} d_{\mathrm{LS}}} \frac{h}{0.57}\left(\frac{R_{E}}{\mathrm{kpc}}\right)^{2} .
$$




\subsection{General Results}

Using the samples mentioned in Section 3, we will try to constrain the free parameters that will satisfy Equation (27). As said before, the information available from the data is the Einstein radius, $R_{E}$, the lens distances $\left(d_{\mathrm{OL}}, d_{\mathrm{LS}}, d_{\mathrm{OS}}\right)$, the lens redshift, and the source redshift. This information is used in the Multinest code (Feroz et al. 2009) to carry out a parameter search for each individual galaxy. We carried out the analysis on the nine galaxies of our subsample of the surveys. Nevertheless, they showed a similar behavior for the range of values of $f_{*}$.

For brevity only representative results are shown, as in Figure 5, for the individual cases of galaxies J0935-0003, J0008-0004, and J220329+020518; these cases include the contribution of the luminous matter to the total mass of the lens as in Equation (27). For the purposes of clarity, in each figure we indicate the radius $r_{s}$ and total mass $M_{s}$ of the soliton profile. We note that the free parameters $r_{\epsilon *}$ and $\alpha_{\mathrm{NFW}}$ appear well constrained if the soliton mass cannot provide the total mass required by the lens system; in the examples shown, this happens if $M_{S}<10^{11.5} M_{\odot}$. Second, the credible regions for the parameters in Figure 5 are in agreement with the theoretical expectations discussed in Section 2.2: there is a minimum value for $r_{\epsilon *}$ due to the constraint imposed by Equation (16), and a maximum value of $\alpha_{\mathrm{NFW}}$ appears due to the maximal contribution of the NFW part of the profile to the total mass in the lens; see also the right panel of Figure 3. Likewise, notice that as $\alpha_{\mathrm{NFW}} \rightarrow 0$ the value of the matching radius $r_{\epsilon *}$ is very well constrained, and this is easily understood from Equation (20): it is $r_{\epsilon *}$ that determines alone the contribution of the NFW part of the profile to the total mass. Indeed, according to our parameterization in Section 2.2, the NFW part of the density profile, under the limit $\alpha_{\mathrm{NFW}} \rightarrow 0$, becomes

$$
\rho_{\mathrm{NFW}}(r)=\frac{\rho_{s} r_{\epsilon *}}{\left(r / r_{s}\right)\left(1+r_{\epsilon *}^{2}\right)^{8}} .
$$

Apart from the presence of $r_{\epsilon *}$ (which in this case is bounded from above, $r_{\epsilon *} \leqslant 1 / \sqrt{15}$; see Equation 17(a)), we also see that the behavior $1 / r$ is the only one that survives from the NFW functional form, and then our results indicate that the outermost behavior $1 / r^{3}$ is left unconstrained.

Finally, observe that the value $\log _{10}\left(M_{s} / M_{\odot}\right)=7.5$ is excluded because the soliton mass $M_{s}$ is so small that the NFW part cannot compensate the required mass for the lens. Recall that there is a matching (continuity) condition for the density profile in which the NFW density $\rho_{\mathrm{NFW}}$ is always smaller than $\rho_{s}$, and this condition makes the NFW part of the profile unable to account for the total mass of the lens even in the limit $\alpha_{\mathrm{NFW}} \rightarrow 0$.

In summary, if the soliton is allowed to provide enough mass to fulfill the matter contribution in the lens, say, $M_{s} \sim 10^{11.5} M_{\odot}$, the analysis will select large values for $r_{\epsilon *}$ so that the NFW tail contribution to the total matter is minimal; see Equation (20). In contrast, if the soliton mass is not large enough, $M_{s}<10^{11.5} M_{\odot}$, it is then possible to find appropriate pairs $\left(\alpha_{\mathrm{NFW}}, r_{\epsilon *}\right)$ for the NFW part of the profile to provide the needed mass for the lens. In this respect, the striped credible regions in Figure 5 represent the degeneracy regions in the plane $\left(\alpha_{\mathrm{NFW}}, r_{\epsilon *}\right)$ for the same mass contribution of the NFW tail to the lens system. Thus, we can see that distinct credible regions can be found for the NFW parameters if the

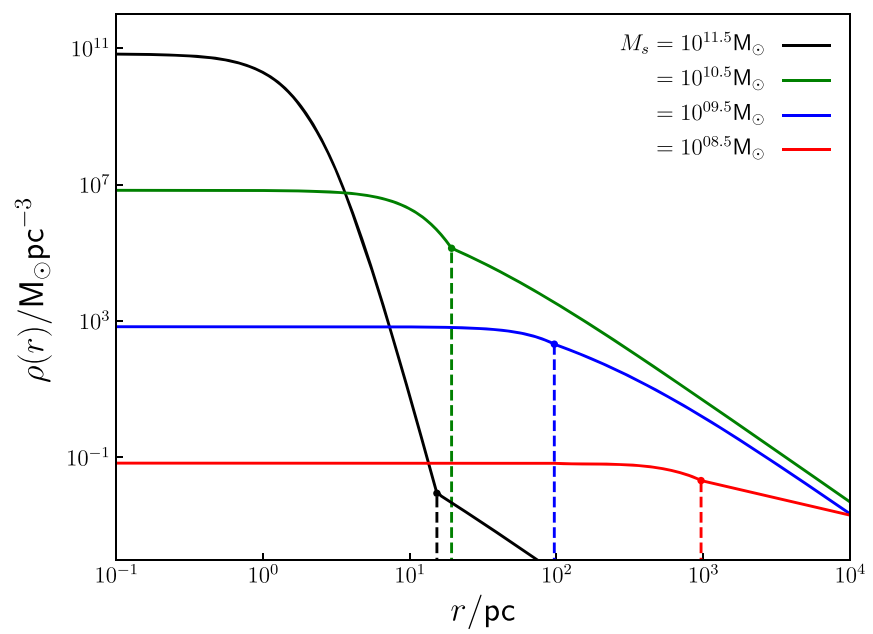

Figure 6. Examples of the density profiles from some of the selected configurations for galaxy J0008-0004 obtained from the constraints in Figure 5. The core region is indicated by the plateau in the curves. Note that the transition to an NFW-like profile happens at larger radii for smaller central density. Shown are cases with the value $\alpha_{\mathrm{NFW}}=1$, except in the case $M_{s}=10^{8.5} M_{\odot}$ (red line $)$, for which $\log \left(\alpha_{\mathrm{NFW}}\right)=-7$.

soliton mass is $10^{8.5}<M_{s} / M_{\odot}<10^{11.5}$, and that the constraints are in agreement with the semianalytic analysis in Section 2.

Another quantity of interest is the resultant density profile of DM in the lens system. Figure 6 shows examples of the density profiles inferred from the posteriors of galaxy J0008-0004 in Figure 5 for a boson mass $m_{a 22}=1$. The soliton core is clearly seen in all curves, and so too is the transition to the NFW part of the profile. The corresponding matching radius $r_{\epsilon}$, in full units, is selected to be at $15.36,19.34,96.94$, and $969.4 \mathrm{pc}$ for the soliton masses $10^{11.5}, 10^{10.5}, 10^{9.5}$, and $10^{8.5} M_{\odot}$, respectively. Not surprisingly, the largest core corresponds to the configuration with the lowest soliton mass for which the matching radius is close to the lower bound suggested in Equation 17(a).

We also report in Figure 7 the results obtained for the lens system J0008-0004 and J220329+020518, for larger or smaller values of the boson mass. For the boson mass $m_{a 22}=10$ we obtain good constraints on the NFW parameters, but the soliton core is very compact in all cases, although a constraint cannot be found if $M_{s}=10^{6.5} M_{\odot}$. For the boson mass of $m_{a 22}=0.1$, we can only obtain well-defined constraints on the NFW parameters when the soliton mass is $M_{s}=10^{10.5} M_{\odot}$, but not for larger or smaller values. Low values of $M_{s}$ imply values of the soliton radius $r_{s}$ that are larger than the Einstein radius, and these kinds of cases are unable to satisfy the lensing constraints. Hence, for a mass of $m_{a 22}=10$, the soliton is much more compact, and it is not by itself adequate to describe a galaxy. But given the fact that the parameters $\alpha_{\mathrm{NFW}}$ and $r_{\epsilon *}$ are also well constrained, we conclude that the lensing effect must be mostly attributed to the NFW part. This is not surprising, as we had already indicated in Section 2.3 that strong lensing could be achieved if $\alpha_{\mathrm{NFW}} \ll 1$. Moreover, a larger boson mass is also in better agreement with recent cosmological constraints (see Iršič et al. 2017) and with estimations based on satellite galaxies of the Milky Way and Andromeda (see Ureña-López et al. 2017).

In contrast, we can see that the constraints become more diffuse if we consider a smaller boson mass of $m_{a 22}=0.1$, although there seems to be some preference for the case in 
Galaxy J0008-0004, $m_{a 22}=10$
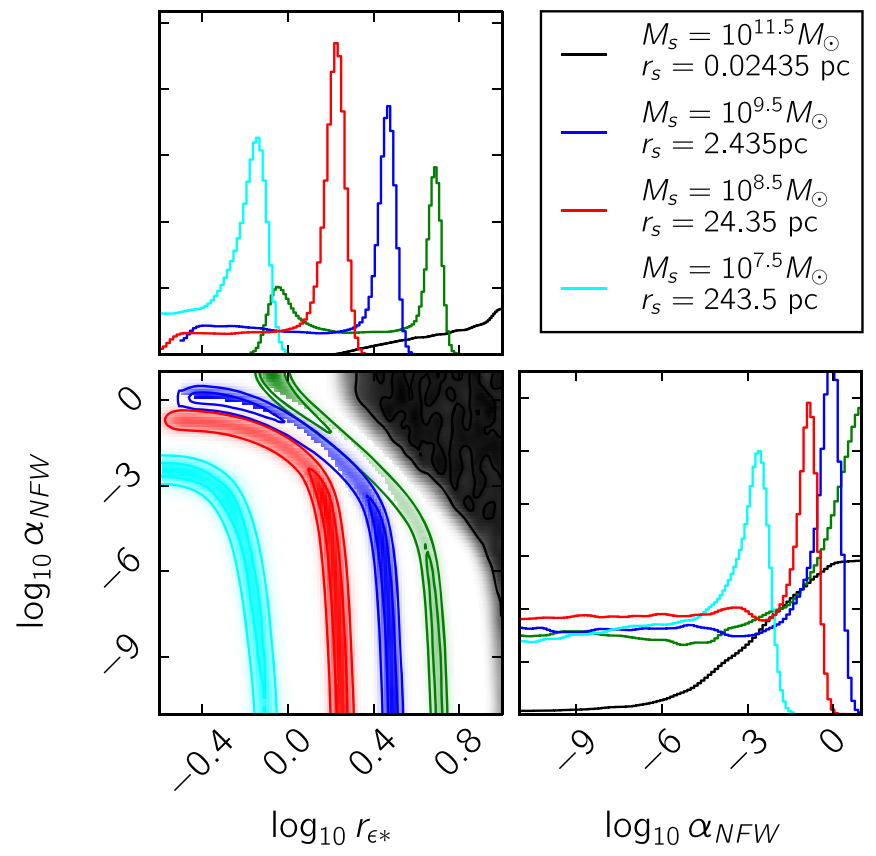

Galaxy J220329+020518, $m_{a 22}=10$
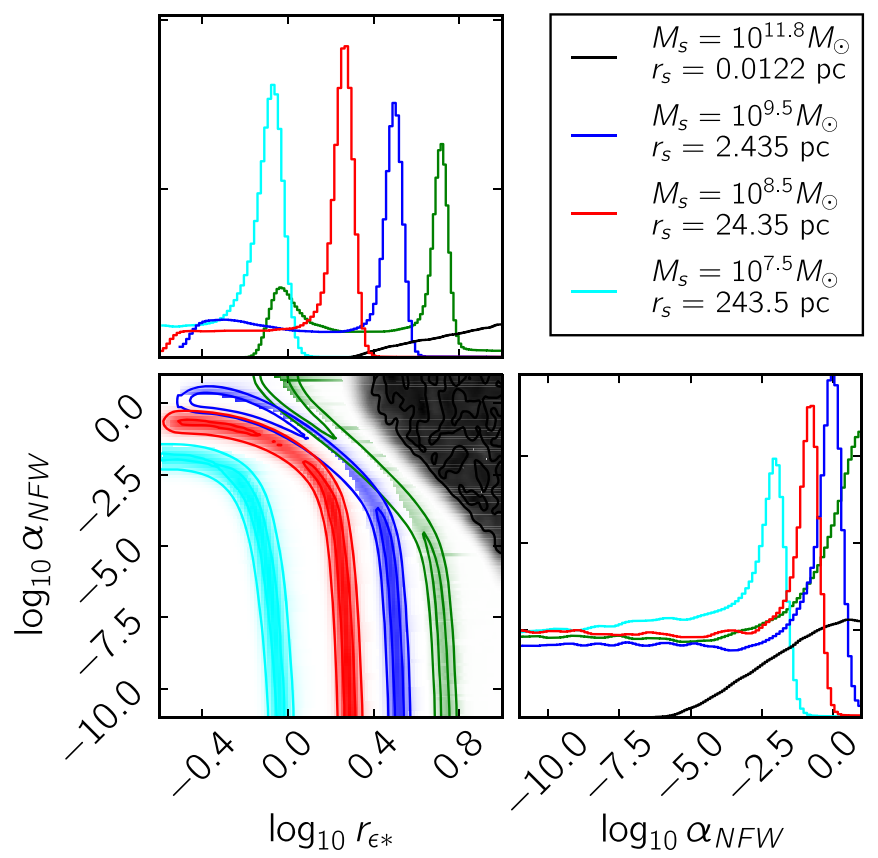

Galaxy J0008-0004, $m_{a 22}=0.1$
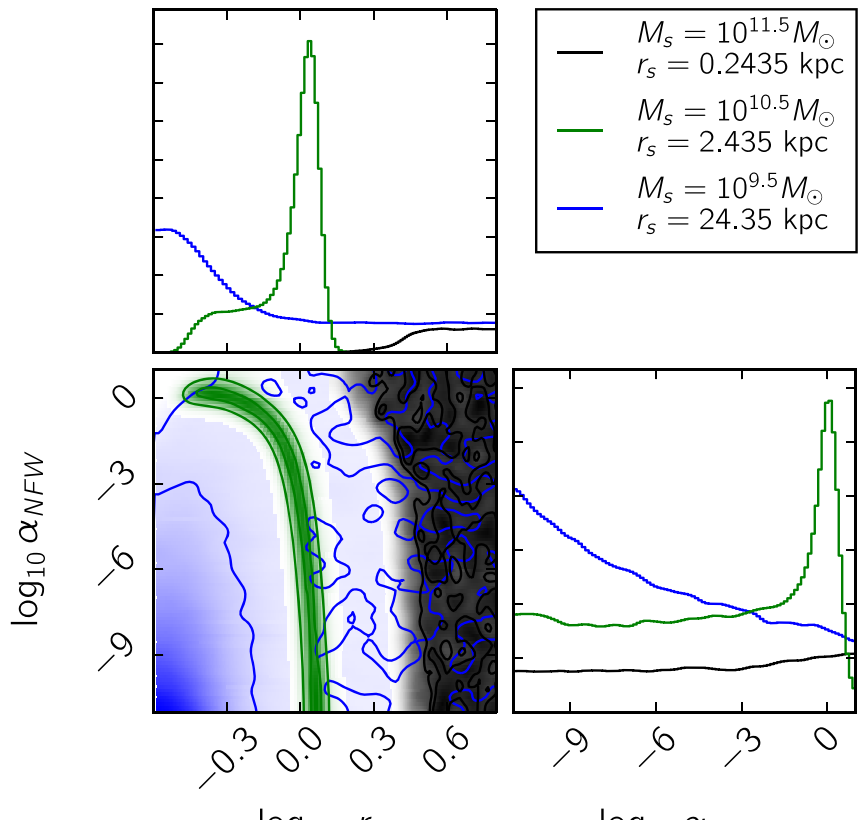

$\log _{10 x} r_{\epsilon *}$

$\log _{10} \alpha_{N F W}$

Galaxy J220329+020518, $m_{a 22}=0.1$
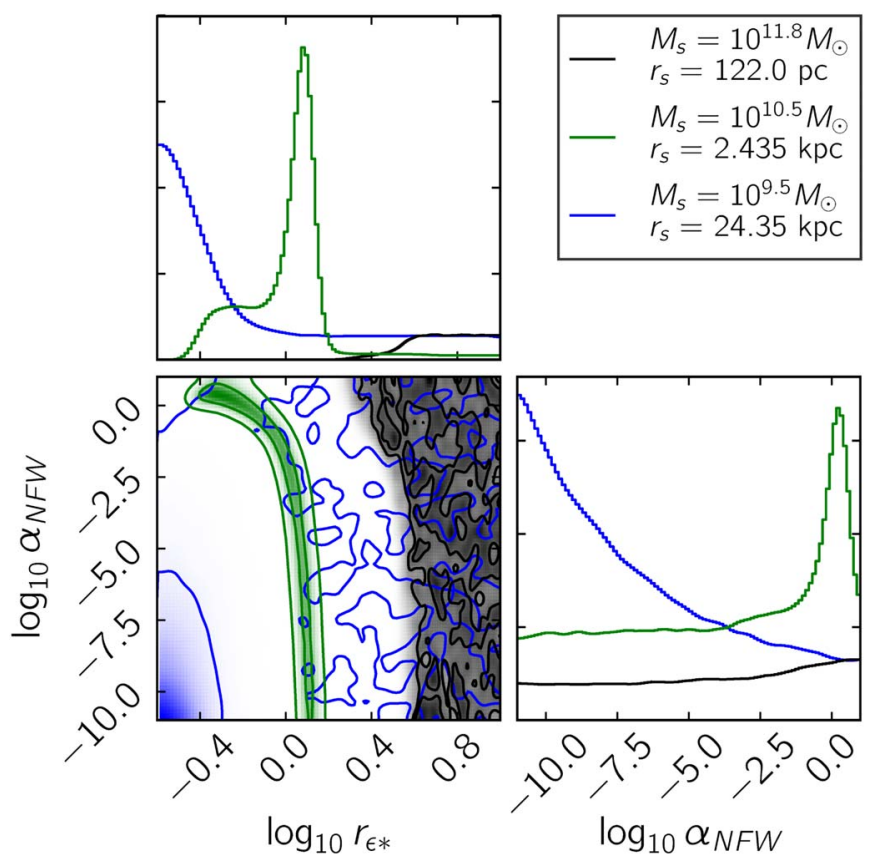

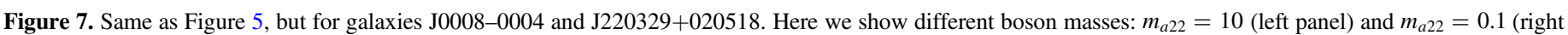
panel). See the text for more details.

which $M_{s}=10^{10.5}$, which also corresponds to a larger soliton radius. This time the resultant configuration would be in agreement with those found in the statistical analysis carried out in González-Morales et al. (2017), which suggests that satellite galaxies put an upper bound on the boson mass that takes the form $m_{a 22}<0.4$.

\section{Conclusions}

We have studied the properties of the so-called WaveDM density profile, assuming that it constitutes the total DM contribution in galaxies for which a gravitational lens has been detected and measured. In doing so, we have adapted the standard lens equations to the particular features of the WaveDM, in that we took into account its soliton core together with its NFW envelope, which is the complete form suggested by numerical simulations of cosmological structure under the WaveDM hypothesis.

We then used the lens equations to make a comparison with actual observations of some lens systems that seem to be DM dominated, although we took into account their baryonic components in a simplified manner. In carrying out the statistical 
analysis, we considered carefully the role of the different free parameters of the WaveDM profile, and in particular the boson mass $m_{a}$, which has to be regarded as a fundamental parameter that should not vary from one galaxy to another.

The overall procedure was then to fix the value of the boson mass and the total mass within the soliton core in the configuration. In consequence, the soliton radius was fixed, and the only free parameters were those of the NFW part of the density profile. In general terms, for large or small values of the boson mass, our results indicate that the soliton structure, if it is as massive as $10^{11.5} M_{\odot}$, is able to fit the measured Einstein radius in the lens systems studied, although this also requires the soliton structure to be extremely compact when compared to the measured scales of the lensing galaxies. This result then indicates that galaxies in general cannot be explained by the soliton structure alone.

Because of the above, we had to consider the complete WaveDM density profile and constrain the NFW free parameters. Generically, and so far for the cases we explored, our analyses suggest that the matching radius for the soliton and NFW parts of the profile is of the same order of magnitude as the soliton radius, $r_{\epsilon} \sim r_{s}$, which is in agreement with the expectation from numerical simulations (e.g., Schwabe et al. 2016; Veltmaat \& Niemeyer 2016; Mocz et al. 2017). In addition, the second free parameter is in general bounded from above as $\alpha_{\mathrm{NFW}}<1$, which just means that the characteristic $\mathrm{NFW}$ radius is larger than the soliton radius, $r_{\mathrm{NFW}}>r_{s}$. Moreover, our results also suggest that the case $\alpha_{\mathrm{NFW}} \rightarrow 0$ is also possible, which in turn means that the density profile decays as $\rho \sim r^{-1}$ at large radii.

On the other hand, for any given value of the boson mass, it was not possible to constrain the NFW parameters in the case where the soliton radius was larger than the Einstein radius, as in such cases the soliton mass is insufficient to produce the required lensing signal. Together with the aforementioned difficulty that the soliton should not provide the whole mass of the lens, we can summarize our results as $M_{s} / M_{\odot}<10^{11.5}$ and $r_{s}<6 \mathrm{kpc}$. Given the similar masses and values of the Einstein radii in the selected sample of galaxies, these constraints can be taken as characteristic of the WaveDM model if the latter is considered to be the DM in them.

By means of Equation (21), the above inequalities can be combined in the following lower bound on the boson mass $m_{a}>10^{-24} \mathrm{eV}$. Notice that this lower bound is in agreement with previous constraints from cosmological and galactic scales (see, e.g., Hlozek et al. 2015; Chen et al. 2017; González-Morales et al. 2016; Ureña-López et al. 2017). Although the lens systems we considered are not able to put strong bounds on the boson mass, they certainly indicate that
As a final note, the lens systems studied here have a subdominant, although non-negligible, baryonic contribution. We expect to extend our analysis to a larger sample considering other surveys with a more detailed and specific description of the baryonic matter contained that could give us better constraints on the soliton features. This is ongoing work that will be presented elsewhere.

L.A.U.-L. wishes to thank Andrew Liddle and the Royal Observatory, Edinburgh, for their kind hospitality in a fruitful sabbatical stay. This work was partially supported by Programa para el Desarrollo Profesional Docente; Dirección de Apoyo a la Investigación y al Posgrado, Universidad de Guanajuato, research Grant 206/2018; Programa Integral de Fortalecimiento Institucional; CONACyT México under grants No. 232893 (sabbatical), 167335, 179881, 269652, 182445, 2286897, 252260 and Fronteras 281; Fundación Marcos Moshinsky; and the Instituto Avanzado de Cosmología Collaboration. This research is supported by The Scottish Universities Physics Alliance and Science and Technology Facilities Council. M. H. is supported by the Science and Technology Facilities Council (Grant No. ST/L000946/1).

\section{Appendix Integral Solutions}

Some useful analytical solutions are given here for the integrals in Equation (18). For the first branch $\theta_{*}<r_{\epsilon *}$, the formula for the first integral is

$$
\begin{aligned}
\int_{0}^{\sqrt{r_{* *}^{2}-\xi_{*}^{2}}} \frac{d z}{\left[1+\alpha_{\mathrm{sol}}^{2} \hat{r}^{2}\right]^{8}} & =\frac{\alpha_{\mathrm{sol}}^{-1}}{\left(1+\alpha_{\mathrm{sol}}^{2} \xi_{*}^{2}\right)^{15 / 2}} \int_{0}^{x} \cos ^{14} u d u, \\
\tan x & =\alpha_{\mathrm{sol}}\left(\frac{r_{\epsilon *}^{2}-\xi_{*}^{2}}{1+\alpha_{\mathrm{sol}}^{2} \xi_{*}^{2}}\right)^{1 / 2},
\end{aligned}
$$

where

$$
\begin{aligned}
\int_{0}^{x} \cos ^{14} u d u= & \frac{429}{2048} x+\frac{1001}{16384}[3 \sin (2 x)+\sin (4 x) \\
& +\frac{1}{3} \sin (6 x)+\frac{1}{11} \sin (8 x)+\frac{1}{55} \sin (10 x) \\
& \left.+\frac{1}{429} \sin (12 x)+\frac{1}{7007} \sin (14 x)\right]
\end{aligned}
$$

whereas for the second integral we obtain

$$
\int_{\sqrt{r_{\epsilon *}^{2}-\xi_{*}^{2}}}^{\infty} \frac{d z}{\hat{r}\left(1+\alpha_{\mathrm{NFW}} \hat{r}\right)^{2}}= \begin{cases}\frac{1}{x^{2}-1}\left(1-\frac{\sqrt{y^{2}-x^{2}}}{1+y}-\frac{2 \operatorname{arctanh}}{\sqrt{1-x^{2}}}\left[\frac{\sqrt{1-x^{2}}}{1+y+\sqrt{y^{2}-x^{2}}}\right]\right) & x<1, \\ \frac{1}{3}\left(1-\frac{y+2}{y+1} \sqrt{\frac{y-1}{y+1}}\right) & x=1, \\ \frac{1}{x^{2}-1}\left(1-\frac{\sqrt{y^{2}-x^{2}}}{1+y}-\frac{2 \arctan }{\sqrt{x^{2}-1}}\left[\frac{\sqrt{x^{2}-1}}{1+y+\sqrt{y^{2}-x^{2}}}\right]\right) & x>1,\end{cases}
$$

most likely a complete WaveDM profile (i.e., comprising a soliton core + NFW tail) is necessary to account for all the diverse observations at galaxy scales. where $x=\alpha_{\mathrm{NFW}} \xi_{*}$ and $y=\alpha_{\mathrm{NFW}} r_{\epsilon *}$. By setting $y=x$, which is equivalent to $r_{\epsilon *}=\xi_{*}$, in Equation (31) we obtain the solution for the second branch in Equation (18). For the case 
$\xi_{*}=0$, which is used in Equation (20), the integral result simply is

$$
\begin{aligned}
\int_{r_{\epsilon *}}^{\infty} \frac{d z}{z\left(1+\alpha_{\mathrm{NFW}} z\right)^{2}}= & \ln \frac{\left(1+\alpha_{\mathrm{NFW}} r_{\epsilon *}\right)}{\alpha_{\mathrm{NFW}} r_{\epsilon *}} \\
& -\frac{1}{\left(1+\alpha_{\mathrm{NFW}} r_{\epsilon *}\right)} .
\end{aligned}
$$

\section{ORCID iDs} Antonio Herrera-Martín (1) https://orcid.org/0000-0002-
3654-4662

Martin Hendry (ํ) https://orcid.org/0000-0001-8322-5405

Alma X. Gonzalez-Morales (i) https://orcid.org/0000-00034089-6924

L. Arturo Ureña-López (ib https://orcid.org/0000-00019752-2830

\section{References}

Adhikari, R., Agostini, M., Drewes, M., et al. 2017, JCAP, 1701, 025 Armengaud, E., Palanque-Delabrouille, N., Marsh, D. J. E., Baur, J., \& Yèche, C. 2017, MNRAS, 471, 4606

Auger, M. W., Treu, T., Bolton, A. S., et al. 2009, ApJ, 705, 1099

Auger, M. W., Treu, T., Bolton, A. S., et al. 2010, ApJ, 724, 511

Bartelmann, M. 1996, A\&A, 313, 697

Bernal, T., Fernández-Hernández, L. M., Matos, T., \& Rodríguez-Meza, M. A. 2018, MNRAS, 475, 1447

Biesiada, M., Piòrkowska, A., \& Malec, B. 2010, MNRAS, 406, 1055

Böhmer, C. G., \& Harko, T. 2007, JCAP, 6, 025

Bolton, A. S., Burles, S., Koopmans, L. V. E., et al. 2008a, ApJ, 682, 964

Bolton, A. S., Burles, S., Koopmans, L. V. E., Treu, T., \& Moustakas, L. A 2006, ApJ, 638, 703

Bolton, A. S., Treu, T., Koopmans, L. V. E., et al. 2008b, ApJ, 684, 248

Boylan-Kolchin, M., Bullock, J. S., \& Kaplinghat, M. 2011, MNRAS, 415, L40

Bullock, J. S., \& Boylan-Kolchin, M. 2017, ARA\&A, 55, 343

Burkert, A. 1995, ApJL, 447, L25

Cao, S., Biesiada, M., Gavazzi, R., Piórkowska, A., \& Zhu, Z.-H. 2015, ApJ, 806, 185

Cao, S., Biesiada, M., Yao, M., \& Zhu, Z.-H. 2016, MNRAS, 461, 2192

Cao, S., Covone, G., \& Zhu, Z.-H. 2012, ApJ, 755, 31

Cao, S., Li, X., Biesiada, M., et al. 2017, ApJ, 835, 92

Cao, S., \& Zhu, Z.-H. 2012, A\&A, 538, A43

Cardone, V. F. 2004, A\&A, 415, 839

Cardone, V. F., Tortora, C., Molinaro, R., \& Salzano, V. 2009, A\&A, 504, 769

Chen, S.-R., Schive, H.-Y., \& Chiueh, T. 2017, MNRAS, 468, 1338

Collett, T. E., Oldham, L. J., Smith, R. J., et al. 2018, Sci, 360, 1342

Cyr-Racine, F.-Y., Sigurdson, K., Zavala, J., et al. 2016, PhRvD, 93, 123527

de Blok, W. J. G. 2010, AdAst, 2010, 789293

Diez-Tejedor, A., Gonzalez-Morales, A. X., \& Profumo, S. 2014, PhRvD, 90, 043517

Feroz, F., Hobson, M. P., \& Bridges, M. 2009, MNRAS, 398, 1601

Futamase, T., \& Yoshida, S. 2001, PThPh, 105, 887

Gavazzi, R., Treu, T., Rhodes, J. D., et al. 2007, ApJ, 667, 176

González-Morales, A. X., Diez-Tejedor, A., Ureña-López, L. A., \& Valenzuela, O. 2013, PhRvD, 87, 021301

González-Morales, A. X., Marsh, D. J. E., Peñarrubia, J., \& Ureña-López, L. 2017, MNRAS, 472, 1346

Gonzalez-Samaniego, A., Avila-Reese, V., \& Colin, P. 2016, ApJ, 819, 101

Goodman, J. 2000, NewA, 5, 103

Grillo, C., Lombardi, M., \& Bertin, G. 2008, A\&A, 477, 397

Guzman, F. S., \& Urena-Lopez, L. A. 2004, PhRvD, 69, 124033

Hlozek, R., Grin, D., Marsh, D. J. E., \& Ferreira, P. G. 2015, PhRvD, 91, 103512

Hogg, D. W. 1999, arXiv:astro-ph/9905116
Hu, W., Barkana, R., \& Gruzinov, A. 2000, PhRvL, 85, 1158

Hui, L., Ostriker, J. P., Tremaine, S., \& Witten, E. 2017, PhRvD, 95, 043541

Iršič, V., Viel, M., Haehnelt, M. G., Bolton, J. S., \& Becker, G. D. 2017, PhRvL, 119, 031302

Jie, A., Bao-Rong, C., \& Li-Xin, X. 2016, ChPhL, 33, 079801

Kaplinghat, M., Tulin, S., \& Yu, H.-B. 2016, PhRvL, 116, 041302

Keeton, C. R. 2001, arXiv:astro-ph/0102341

Klypin, A. A., Kravtsov, A. V., Valenzuela, O., \& Prada, F. 1999, ApJ, 522, 82

Kochanek, C. S. 1991, ApJ, 373, 354

Kochanek, C. S., Keeton, C. R., \& McLeod, B. A. 2001, ApJ, 547, 50

Koopmans, L. V. E. 2005, MNRAS, 363, 1136

Koopmans, L. V. E., Treu, T., Bolton, A. S., Burles, S., \& Moustakas, L. A. 2006, ApJ, 649, 599

Lee, J.-W. 2018, EPJWC, 168, 06005

Linares Cedeño, F. X., Gonzalez-Morales, A. X., \& Arturo Ureña-López, L. 2017, PhRvD, 96, 061301

Lyskova, N., Churazov, E., \& Naab, T. 2018, MNRAS, 475, 2403

Maccio, A. V., Paduroiu, S., Anderhalden, D., Schneider, A., \& Moore, B. 2012, MNRAS, 424, 1105

Marsh, D. J. E., \& Pop, A.-R. 2015, MNRAS, 451, 2479

Matos, T., Guzman, F. S., \& Urena-Lopez, L. A. 2000, CQGra, 17, 1707

Matos, T., \& Urena-Lopez, L. A. 2001, PhRvD, 63, 063506

Mitchell, J. L., Keeton, C. R., Frieman, J. A., \& Sheth, R. K. 2005, ApJ, 622,81

Mocz, P., Vogelsberger, M., Robles, V., et al. 2017, MNRAS, 471, 4559

Mollerach, S., \& Roulet, E. 2002, Gravitational Lensing and Microlensing (Singapore: World Scientific)

Muñoz, J. A., Kochanek, C. S., \& Keeton, C. R. 2001, ApJ, 558, 657

Nair, S., \& Garrett, M. A. 1996, in Astrophysical Applications of Gravitational, ed. C. S. Kochanek \& J. N. Hewitt (Dordrecht: Springer), 195

Navarro, J. F., Frenk, C. S., \& White, S. D. M. 1997, ApJ, 490, 493

Newton, E. R., Marshall, P. J., Treu, T., et al. 2011, ApJ, 734, 104

Niikura, H., Takada, M., Okabe, N., Martino, R., \& Takahashi, R. 2015, PASJ, 67, 103

Ofek, E. O., Maoz, D., Rix, H.-W., Kochanek, C. S., \& Falco, E. E. 2006, ApJ, 641, 70

Ofek, E. O., Rix, H.-W., \& Maoz, D. 2003, MNRAS, 343, 639

Oh, S.-H., de Blok, W. J. G., Brinks, E., Walter, F., \& Kennicutt, J. R. C. 2011, AJ, 141, 193

Park, Y., \& Ferguson, H. C. 2003, ApJL, 589, L65

Planck Collaboration, Ade, P. A. R., Aghanim, N., et al. 2015, A\&A, 594, A13

Planck Collaboration, Ade, P. A. R., Aghanim, N., et al. 2016, A\&A, 594, A13

Robles, V. H., \& Matos, T. 2013, PhRvD, 88, 083008

Ruffini, R., \& Bonazzola, S. 1969, PhRv, 187, 1767

Sawala, T., Guo, Q., Scannapieco, C., Jenkins, A., \& White, S. 2011, MNRAS, 413,659

Schive, H.-Y., \& Chiueh, T. 2018, MNRAS, 473, 36

Schive, H.-Y., Chiueh, T., \& Broadhurst, T. 2014a, NatPh, 10, 496

Schive, H.-Y., Liao, M.-H., Woo, T.-P., et al. 2014b, PhRvL, 113, 261302

Schneider, P., Ehlers, J., \& Falco, E. 1999, Gravitational Lenses, Astronomy and Astrophysics Library (Berlin: Springer)

Schwabe, B., Niemeyer, J. C., \& Engels, J. F. 2016, PhRvD, 94, 043513

Sereno, M. 2002, A\&A, 393, 757

Sonnenfeld, A., Gavazzi, R., Suyu, S. H., Treu, T., \& Marshall, P. J. 2013a, ApJ, 777, 97

Sonnenfeld, A., Treu, T., Gavazzi, R., et al. 2013b, ApJ, 777, 98

Suárez, A., Robles, V. H., \& Matos, T. 2014, in Accelerated Cosmic Expansion, Vol. 38, ed. C. Moreno González, J. E. Madriz Aguilar, \& L. M. Reyes Barrera, 107

Suyu, S. H., Treu, T., Hilbert, S., et al. 2014, ApJL, 788, L35

Tortora, C., Napolitano, N. R., Roy, N., et al. 2018, MNRAS, 473, 969

Treu, T., \& Koopmans, L. V. E. 2004, ApJ, 611, 739

Trick, W. H., van de Ven, G., \& Dutton, A. A. 2016, MNRAS, 463, 3151

Ureña-López, L. A., \& Gonzalez-Morales, A. X. 2016, JCAP, 1607, 048

Ureña-López, L. A., Robles, V. H., \& Matos, T. 2017, PhRvD, 96, 043005

Veltmaat, J., \& Niemeyer, J. C. 2016, PhRvD, 94, 123523

Winitzki, S. 2008, A Handy Approximation for the Error Function and its Inverse, http://www.academia.edu/9730974/A_handy_approximation_ for_the_error_function_and_its_inverse

Wright, C. O., \& Brainerd, T. G. 2000, ApJ, 534, 34

Wyithe, J. S. B., Turner, E. L., \& Spergel, D. N. 2001, ApJ, 555, 504

Zhang, U.-H., \& Chiueh, T. 2017a, PhRvD, 96, 063522

Zhang, U.-H., \& Chiueh, T. 2017b, PhRvD, 96, 023507 